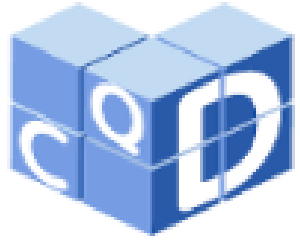

Revista Eletrônica

Paulista de Matemática

ISSN 2316-9664

Volume 16, dez. 2019

Francisco Regis Vieira Alves Instituto Federal de Educação, Ciência e Tecnologia do Estado do Ceará - IFCE

fregis@ifce.edu.br

\section{Sequência de Oresme e algumas propriedades (matriciais) generalizadas}

The Oresme sequence and some (matrix) generalized properties

\section{Resumo}

O presente trabalho aborda algumas propriedades sobre os números de Oresme, sobre os k-números de Oresme, os polinômios de Oresme e, a partir desses, introduz novas entidades matemáticas chamados de Quaternions, Octônions e Sedenions de Oresme. Algumas das propriedades ainda não discutidas na literatura constituem o resultado principal do trabalho envolvendo inesperadas propriedades algébricas das representações matriciais de segunda ordem.

Palavras-chave: Números de Oresme, k-números de Oresme, Quaternions e Octônions de Oresme, Sedênions de Oresme.

\begin{abstract}
The present work deals with some properties of the Oresme numbers, the Oresme k-numbers, the Oresme polynomials and, from these, introduces new mathematical entities called Oresme Quaternions, Octônions and Sedenions. Some of the properties not yet discussed in the literature are the main result of the work involving unexpected algebraic properties of the second orther matrix representations.
\end{abstract}

Keywords: Oresme numbers, k-Oresme numbers, Oresme Quaternions and Octonios, Oresme Sedenions. 


\section{Introdução}

No presente trabalho discutiremos algumas propriedades sobre os números de Oresme e a sequência de Oresme. Evidenciaremos que alguns trabalhos proporcionam a compreensão sobre seu processo de generalização, inclusive, a partir da verificação de resultados recentes que confirmam um interesse atual por parte de especialistas. Por outro lado, a introdução dos instrumentos conceituais da Álgebra Linear permitiu um amplo avanço no estudo de propriedades mais gerais sobre a Teoria das Sequências Recorrentes Homogêneas de ordem 'n' (KAUERS; PAULE, 2010). Assim, evidenciaremos que determinadas propriedades matriciais permitem uma elegante representação para os Quaternions, Octônions e Sedênions de Oresme que ensejamos apresentar na última seção e constitui o resultado mais importante do presente escrito.

\section{Os números de Oresme e a Sequência de Oresme}

Horadam (1974) comenta que na metade do século XIV o clérigo Nicole Oresme (1320 1382) encontrou a soma dos seguintes números racionais que indicamos em seguida $\frac{1}{2}, \frac{2}{4}, \frac{3}{8}, \frac{4}{16}, \frac{5}{32}, \frac{6}{64}, \frac{7}{128}, \frac{8}{256}, \frac{9}{512}, \cdots, \frac{n}{2^{n}}, \cdots$. O autor acrescenta que o Oresme não publicou nenhum desses resultados. Ademais, a referida sequência enigmática possui um caráter, também, de interesse na Biologia, na medida em que, a partir dos dois primeiros termos, podemos estimar a quantidade de pais, avós e determinar tal proporção em qualquer geração. Stillwell $(1989$, p. 119) comenta que a descoberta do comportamento de convergência da série indicada pela soma infinita $\sum_{i=0}^{\infty} O_{i}=\frac{1}{2}+\frac{2}{4}+\frac{3}{8}+\frac{4}{16}+\frac{5}{32}+\frac{6}{64}+\frac{7}{128}+\frac{8}{256}+\cdots+\frac{n}{2^{n}}+\cdots=2$ foi devida a Nicole Oresme, que empregou ideias primitivas ao que hoje conhecemos como a integral imprópria (NICODEMI, 2010). Antes de prosseguirmos, vejamos a seguinte definição fundamental.

Definição 1: A sequência de números $\left\{O_{n}\right\}_{n \in I N}=\left\{\frac{n}{2^{n}}\right\}_{n \in I N}$ é descrita pela seguinte relação de recorrência $O_{n+2}=O_{n+1}-\frac{1}{4} O_{n}, O_{0}=0, O_{1}=\frac{1}{2}, n \geq 0$ (HORADAM, 1974).

Horadam (1974, p. 267) explica que "a sequência de Oresme pode ser extendida para o lado esquerdo, incluindo números negativos, se observarmos o comportamento padrão de tal sequência”. Na figura abaixo observamos um diagrama simplificado proposto por Horadam (1974) sobre a descrição explícita dos termos do tipo indicado no conjunto $\left\{O_{-n}\right\}_{n \in I N}$.

$$
\begin{array}{lllllllllllllllllll}
\cdots & 0_{-7} & O_{-6} & O_{-5} & O_{-4} & O_{-3} & 0_{-2} & O_{-1} & O_{0} & O_{1} & O_{2} & O_{3} & O_{4} & O_{5} & O_{6} & O_{7} & \cdots \\
\cdots-896 & -384 & -160 & -64 & -24 & -8 & -2 & 0 & \frac{1}{2} & \frac{2}{4} & \frac{3}{8} & \frac{4}{16} & \frac{5}{32} & \frac{6}{64} & \frac{7}{128} & \cdots
\end{array}
$$

Figura 1. Horadam (1974) explica a descrição do modelo de expansão da sequência de Oresme segundo índices inteiros negativos e elementos do tipo $O_{-n}$. 
Mais recentemente, Cook (2004) fornece novas identidades envolvendo tais números, tais como: $O_{n+2}-\frac{3}{4} O_{n}+\frac{1}{4} O_{n-1}=0, O_{n+2}-\frac{3}{4} O_{n+1}+\frac{1}{16} O_{n-1}=0, \sum_{i=0}^{n-1} O_{i}=4\left(\frac{1}{2}-O_{n+1}\right), \sum_{i=0}^{n-1}(-1)^{i} O_{i}$ $=\frac{4}{5}\left[-\frac{1}{2}+(-1)^{n}\left(O_{n+1}-2 O_{n}\right)\right], \sum_{i=0}^{n-1} O_{2 i}=\frac{4}{9}\left[2+O_{2 n-1}-5 O_{2 n}\right], \sum_{i=0}^{n-1} O_{2 i+1}=\frac{1}{9}\left[10+5 O_{2 n-1}-16 O_{2 n}\right]$.

Por outro lado, a partir da definição 1 podemos determinar precisamente os elementos com índices inteiros negativos. De fato, a partir da definição de recorrência $O_{n+2}=O_{n+1}-\frac{1}{4} O_{n}$ podemos assumir que $n=-1$, portanto, segue $O_{1}=O_{0}-\frac{1}{4} O_{-1} \leftrightarrow O_{-1}=-2$. Repetindo o procedimento anterior, tomamos $n=-2$ e, portanto $O_{0}=O_{-1}-\frac{1}{4} O_{-2} \leftrightarrow O_{-2}=-8$. Horadam $(1974, \mathrm{p}$. 268) comenta, sem muitos pormenores, que podemos estabelecer que $O_{n}=\left\{\begin{array}{l}\frac{n}{2^{n}}, n \geq 0 \\ -n \cdot 2^{n}, n<0\end{array}\right.$. Isto se deve ao fato de que as propriedades obtidas por Horadam são imediatas, a partir da definição da sequência recorrente. Dessa forma, podemos determinar os números de Oresme da forma $O_{n}=n \cdot 2^{n}$, com $n$ inteiro. Em um contexto mais geral, Horadam (1974) aborda o modelo geral que indicamos por $W_{n+2}=p \cdot W_{n+1}-q \cdot W_{n}, W_{0}=a, W_{1}=b$, onde os números são inicialmente inteiros $p, q$ e arbritrários. Assim, Horadam (1974) que para a extensão de tal modelo, requer que os números $p, q$ sejam racionais. De sorte que, para determinar os elementos do conjunto $\left\{O_{n}\right\}_{n \in I N}=\left\{\frac{n}{2^{n}}\right\}_{n \in I N}$ requer assumir particularmente que $p=1, q=\frac{1}{2}, a=0, b=1$. Simbolicamente, lidamos com a seguinte sequência $W_{n}\left(0, \frac{1}{2} ; 1, \frac{1}{2}\right)=O_{n}\left(0, \frac{1}{2} ; 1, \frac{1}{2}\right)$.

Por outro lado, mais recentemente, registramos a introdução na literatura especializada da seguinte definição que representa, como esclarece Cook (2004, p. 95), uma das várias formas ou maneiras de sua generalização. Cook (2004) apresenta a seguinte recorrência mais geral $A_{k, n+2}=A_{k, n+1}-\frac{k-1}{k^{2}} A_{k, n}-\frac{k-2}{k^{n+2}}, k \geq 2$ e, em seguida, apresenta a definição dos números k de Oresme. Por exemplo, se tomarmos o termo da forma $A_{k, n}=\frac{n}{k^{n}}, k \geq 2$, como explica Cook (2004, p. 95), devemos encontrar a seguinte relação de recorrência correspondente como indicamos logo em seguida na definição 2.

Definição 2: Para todo inteiro $k \geq 2$ os números do tipo $\left\{O_{k, n}\right\}_{n \in I N}$ são definidos pela relação homogênea de recorrência $O_{k, n+2}=O_{k, n+1}-\frac{1}{k^{2}} O_{k, n}, O_{k, 0}=0, O_{k, 1}=\frac{1}{k}$.

Vejamos alguns de seus elementos que indicamos: $O_{k, 2}=O_{k, 1}-\frac{1}{k^{2}} O_{k, 0}=\frac{1}{k}, O_{k, 3}=\frac{k^{2}-1}{k^{3}}$, 
$O_{k, 4}=\frac{k^{2}-2}{k^{3}}, \quad O_{k, 5}=\frac{k^{4}-3 k^{2}+1}{k^{5}}, \quad O_{k, 6}=\frac{k^{4}-4 k^{2}+3}{k^{5}}, \quad O_{k, 7}=\frac{k^{6}-5 k^{4}+6 k^{2}-1}{k^{7}}$,

$O_{k, 8}=\frac{k^{6}-5 k^{4}+6 k^{2}-1}{k^{7}}, O_{k, 9}=\frac{k^{8}-7 k^{6}+15 k^{4}-10 k^{2}+1}{k^{9}}, O_{k, 10}=\frac{k^{8}-8 k^{6}+21 k^{4}-20 k^{2}+5}{k^{9}}$,

$O_{k, 11}=\frac{k^{10}-9 k^{8}+28 k^{6}-35 k^{4}+15 k^{2}-1}{k^{11}}$, etc. Por outro lado, podemos ainda verificar que:

$O_{k,-1}=k^{2}\left(O_{k, 0}-O_{k, 1}\right)=-k, \quad O_{k,-2}=-k^{3}, \quad O_{k,-3}=-k^{3}\left(k^{2}-1\right), \quad O_{k,-4}=-k^{5}\left(k^{2}-2\right)$,

$O_{k,-5}=-k^{5}\left(k^{4}-3 k^{2}+1\right), O_{k,-6}=-k^{7}\left(k^{4}-4 k^{2}+3\right), O_{k,-7}=-k^{7}\left(k^{6}-5 k^{4}+6 k^{2}-1\right)$,

$O_{k,-8}=-k^{9}\left(k^{6}-6 k^{4}+10 k^{2}-4\right), \quad O_{k,-9}=-k^{9}\left(k^{8}-7 k^{6}+15 k^{4}-10 k^{2}+1\right)$,

$O_{k,-10}=-k^{11}\left(k^{8}-8 k^{6}+21 k^{4}-20 k^{2}+5\right)$, etc.

Os números denotados por $\left\{O_{k, n}\right\}_{n \in I N}$ são nominados por Cerda-Morales (2019) como os números k-Oresme. Observamos, todavia, a possibilidade análoga de determinação dos elementos do conjunto $\left\{O_{k,-n}\right\}_{n \in I N}$. Empregando, assim, um raciocínio semelhante ao de Horadam (1974), podemos definir os k-Números de Oresme da forma $O_{k, n}=\left\{\begin{array}{l}\frac{n}{k^{n}}, n \geq 0 \\ -n \cdot k^{n}, n<0\end{array}\right.$, com $k \geq 2$.

Mais recentemente, encontramos no trabalho de Cerda-Morales (2019) a proposição das matrizes associadas por $M=\left(\begin{array}{cc}1 & -\frac{1}{4} \\ 1 & 0\end{array}\right), M_{k}=\left(\begin{array}{cc}1 & -\frac{1}{k^{2}} \\ 1 & 0\end{array}\right)$ e, a vamos examinar as seguintes potências matriciais especiais: $M^{1}=\left(\begin{array}{cc}1 & -\frac{1}{4} \\ 1 & 0\end{array}\right)=\left(\begin{array}{cc}2\left(\frac{1}{2}\right) & -\frac{1}{2}\left(\frac{1}{2}\right) \\ 2\left(\frac{1}{2}\right) & -\frac{1}{2}(0)\end{array}\right)=\left(\begin{array}{cc}2 O_{2} & -\frac{1}{2} O_{1} \\ 2 O_{1} & -\frac{1}{2} O_{0}\end{array}\right)$, $M^{2}=\left(\begin{array}{ll}\frac{3}{4} & -\frac{1}{4} \\ 1 & -\frac{1}{4}\end{array}\right)=\left(\begin{array}{ll}2\left(\frac{3}{8}\right) & -\frac{1}{2}\left(\frac{2}{4}\right) \\ 2\left(\frac{2}{4}\right) & -\frac{1}{2}\left(\frac{1}{2}\right)\end{array}\right)=\left(\begin{array}{cc}2 O_{3} & -\frac{1}{2} O_{2} \\ 2 O_{2} & -\frac{1}{2} O_{1}\end{array}\right), M^{3}=\left(\begin{array}{cc}\frac{1}{2} & -\frac{3}{16} \\ \frac{3}{4} & -\frac{1}{4}\end{array}\right)=$ $\left(\begin{array}{ll}2\left(\frac{4}{16}\right) & -\frac{1}{2}\left(\frac{3}{8}\right) \\ 2\left(\frac{3}{8}\right) & -\frac{1}{2}\left(\frac{2}{4}\right)\end{array}\right)=\left(\begin{array}{cc}2 O_{4} & -\frac{1}{2} O_{3} \\ 2 O_{3} & -\frac{1}{2} O_{2}\end{array}\right), M^{4}=\left(\begin{array}{cc}\frac{5}{16} & -\frac{1}{8} \\ \frac{1}{2} & -\frac{3}{16}\end{array}\right)=\left(\begin{array}{cc}2\left(\frac{5}{32}\right) & -\frac{1}{2}\left(\frac{4}{16}\right) \\ 2\left(\frac{4}{16}\right) & -\frac{1}{2}\left(\frac{3}{8}\right)\end{array}\right)=\left(\begin{array}{cc}2 O_{5} & -\frac{1}{2} O_{4} \\ 2 O_{4} & -\frac{1}{2} O_{3}\end{array}\right)$,

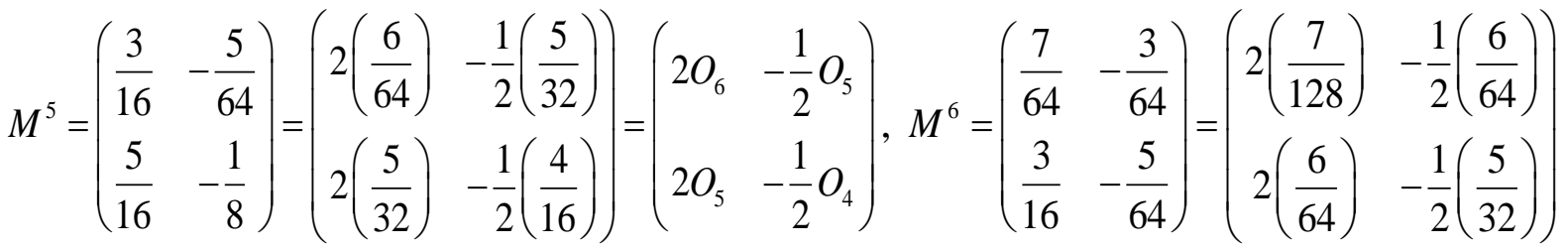


$=\left(\begin{array}{cc}2 O_{7} & -\frac{1}{2} O_{6} \\ 2 O_{6} & -\frac{1}{2} O_{5}\end{array}\right), M^{7}=\left(\begin{array}{cc}\frac{1}{16} & -\frac{7}{256} \\ \frac{7}{64} & -\frac{3}{64}\end{array}\right)=\left(\begin{array}{cc}2\left(\frac{8}{256}\right) & -\frac{1}{2}\left(\frac{7}{128}\right) \\ 2\left(\frac{7}{128}\right) & -\frac{1}{2}\left(\frac{6}{64}\right)\end{array}\right)=\left(\begin{array}{cc}2 O_{8} & -\frac{1}{2} O_{7} \\ 2 O_{7} & -\frac{1}{2} O_{6}\end{array}\right)$. Por outro lado, podemos ainda verificar que se tem $M^{-1}=\left(\begin{array}{cc}0 & 1 \\ -4 & 4\end{array}\right)=\left(\begin{array}{cc}2(0) & -\frac{1}{2}(-2) \\ 2(-2) & -\frac{1}{2}(-8)\end{array}\right)=\left(\begin{array}{cc}2 O_{0} & -\frac{1}{2} O_{-1} \\ 2 O_{-1} & -\frac{1}{2} O_{-2}\end{array}\right)$, $M^{-2}=\left(\begin{array}{cc}-4 & 4 \\ -16 & 12\end{array}\right)=\left(\begin{array}{ll}2(-2) & -\frac{1}{2}(-8) \\ 2(-8) & -\frac{1}{2}(-24)\end{array}\right)=\left(\begin{array}{cc}2 O_{-1} & -\frac{1}{2} O_{-2} \\ 2 O_{-2} & -\frac{1}{2} O_{-3}\end{array}\right), M^{-3}=\left(\begin{array}{ll}-16 & 12 \\ -48 & 32\end{array}\right)=\left(\begin{array}{cc}2(-8) & -\frac{1}{2}(-24) \\ 2(-24) & -\frac{1}{2}(-64)\end{array}\right)$ $=\left(\begin{array}{cc}2 O_{-2} & -\frac{1}{2} O_{-3} \\ 2 O_{-3} & -\frac{1}{2} O_{-4}\end{array}\right), M^{-4}=\left(\begin{array}{cc}-48 & 32 \\ -128 & 80\end{array}\right)=\left(\begin{array}{cc}2(-24) & -\frac{1}{2}(-64) \\ 2(-64) & -\frac{1}{2}(-160)\end{array}\right)=\left(\begin{array}{cc}2 O_{-3} & -\frac{1}{2} O_{-4} \\ 2 O_{-4} & -\frac{1}{2} O_{-5}\end{array}\right), M^{-5}=$ $\left(\begin{array}{cc}-128 & 80 \\ -320 & 192\end{array}\right)=\left(\begin{array}{cc}2 O_{-4} & -\frac{1}{2} O_{-5} \\ 2 O_{-5} & -\frac{1}{2} O_{-6}\end{array}\right), M^{-6}=\left(\begin{array}{cc}-320 & 192 \\ -768 & 448\end{array}\right)=\left(\begin{array}{ll}2(-160) & -\frac{1}{2}(-384) \\ 2(-384) & -\frac{1}{2}(-896)\end{array}\right)=\left(\begin{array}{cc}2 O_{-5} & -\frac{1}{2} O_{-6} \\ 2 O_{-6} & -\frac{1}{2} O_{-7}\end{array}\right)$, etc. Vamos definir, dessa forma, as seguintes matrizes especiais $M(n)=\left(\begin{array}{cc}2 O_{n+1} & -\frac{1}{2} O_{n} \\ 2 O_{n} & -\frac{1}{2} O_{n-1}\end{array}\right)$, $M_{k}(n)=\left(\begin{array}{cc}k \cdot O_{k, n+1} & -\frac{1}{k} O_{k, n} \\ k \cdot O_{k, n} & -\frac{1}{k} O_{k, n-1}\end{array}\right)$, com $k \geq 2$. Com origem nesses casos preliminares vejamos o seguinte teorema inicial do presente trabalho.

Teorema 1: Para todo inteiro $n \geq 0$ temos: (i) $M(n)=M^{n}=\left(\begin{array}{cc}1 & -\frac{1}{4} \\ 1 & 0\end{array}\right)^{n}=\left(\begin{array}{cc}2 O_{n+1} & -\frac{1}{2} O_{n} \\ 2 O_{n} & -\frac{1}{2} O_{n-1}\end{array}\right)$, $M_{k}(n)=\left(\begin{array}{cc}1 & -\frac{1}{k^{2}} \\ 1 & 0\end{array}\right)^{n}=\left(\begin{array}{cc}2 O_{k, n+1} & -\frac{1}{2} O_{k, n} \\ 2 O_{k, n} & -\frac{1}{2} O_{k, n-1}\end{array}\right)$; (ii) $M(-n)=\left(\begin{array}{cc}1 & -\frac{1}{4} \\ 1 & 0\end{array}\right)^{-n}=(M)^{-n}=\left(\begin{array}{cc}2 O_{-n+1} & -\frac{1}{2} O_{-n} \\ 2 O_{-n} & -\frac{1}{2} O_{-n-1}\end{array}\right)$ e $M_{k}(-n)=\left(M_{k}(n)\right)^{-1}$, para todo inteiro $k \geq 2$; (iii) $O_{n-1} O_{n+1}-O_{n}^{2}=-\left(-\frac{1}{4}\right)^{n}=-\frac{1}{\left(-2^{2 n}\right)}$; (iv) $O_{-n-1} O_{-n+1}-O_{-n}^{2}=-\left(-\frac{1}{4}\right)^{-n} ;$ (v) $O_{m+n}=2 O_{m} O_{n+1}-\frac{1}{2} O_{m-1} O_{n} ;(\mathrm{vi}) O_{-(m+n)}=2 O_{-m} O_{-n+1}-\frac{1}{2} O_{-m-1} O_{-n}$ 
; (vi) $M(m+n)=M(m) \cdot M(n), \quad M_{k}(m+n)=M_{k}(m) \cdot M_{k}(n), M(-m-n)=M(-m) \cdot M(-n)$, $M_{k}(-m-n)=M_{k}(-m)_{k} \cdot M(-n)$.

Prova. Por indução matemática, já vimos que $M^{1}=\left(\begin{array}{cc}2 O_{2} & -\frac{1}{2} O_{1} \\ 2 O_{1} & -\frac{1}{2} O_{0}\end{array}\right)$, No passo indutivo, vejamos que $M(n)=\left(\begin{array}{cc}2 O_{n+1} & -\frac{1}{2} O_{n} \\ 2 O_{n} & -\frac{1}{2} O_{n-1}\end{array}\right)$. Em seguida, vamos considerar a seguinte potência da matriz $M^{n+1}=M^{n} M=\left(\begin{array}{cc}2 O_{n+1} & -\frac{1}{2} O_{n} \\ 2 O_{n} & -\frac{1}{2} O_{n-1}\end{array}\right)\left(\begin{array}{cc}1 & -\frac{1}{4} \\ 1 & 0\end{array}\right)=\left(\begin{array}{cc}2 O_{n+1}-\frac{1}{2} O_{n} & -\frac{1}{2} O_{n+1} \\ 2 O_{n}-\frac{1}{2} O_{n-1} & -\frac{1}{2} O_{n}\end{array}\right)=\left(\begin{array}{cc}2\left(O_{n+1}-\frac{1}{4} O_{n}\right) & -\frac{1}{2} O_{n+1} \\ 2\left(O_{n}-\frac{1}{4} O_{n-1}\right) & -\frac{1}{2} O_{n}\end{array}\right)=$ $=\left(\begin{array}{cc}2 O_{n+2} & -\frac{1}{2} O_{n+1} \\ 2 O_{n+1} & -\frac{1}{2} O_{n}\end{array}\right)$. Com origem no item $M(n)=M^{n}=\left(\begin{array}{cc}2 O_{n+1} & -\frac{1}{2} O_{n} \\ 2 O_{n} & -\frac{1}{2} O_{n-1}\end{array}\right)$ verificamos que $\operatorname{det}\left(\begin{array}{cc}1 & -\frac{1}{4} \\ 1 & 0\end{array}\right)^{n}=-O_{n-1} O_{n+1}+O_{n}^{2}=\left(\frac{1}{4}\right)^{n}=\frac{1}{4^{n}}$. Por outro lado, de acordo com Horadam (1974), podemos usar a descrição dos números de Oresme da forma $O_{n}=\left\{\begin{array}{l}\frac{n}{2^{n}}, n \geq 0 \\ -n \cdot 2^{n}, n<0\end{array}\right.$. Tomaremos a matriz $M(-n)=\left(\begin{array}{cc}2 O_{-n+1} & -\frac{1}{2} O_{-n} \\ 2 O_{-n} & -\frac{1}{2} O_{-n-1}\end{array}\right)=\left(\begin{array}{cc}2 O_{-(n-1)} & -\frac{1}{2} O_{-n} \\ 2 O_{-n} & -\frac{1}{2} O_{-(n+1)}\end{array}\right)=\left(\begin{array}{cc}2\left(-(n-1) \cdot 2^{n-1}\right) & -\frac{1}{2}\left(-n \cdot 2^{n}\right) \\ 2\left(-n \cdot 2^{n}\right) & -\frac{1}{2}\left(-(n+1) \cdot 2^{n+1}\right)\end{array}\right)=$ $=\left(\begin{array}{cc}-(n-1) \cdot 2^{n} & \frac{n \cdot 2^{n}}{2} \\ -n \cdot 2^{n+1} & (n+1) \cdot 2^{n}\end{array}\right)=\left(2^{n}\right) \cdot\left(\begin{array}{cc}-(n-1) & \frac{n}{2} \\ -2 n & (n+1)\end{array}\right)=\left(2^{2 n}\right) \cdot\left(\begin{array}{cc}\frac{-(n-1)}{2^{n}} & \frac{n}{2^{n+1}} \\ \frac{-2 n}{2^{n}} & \frac{(n+1)}{2^{n}}\end{array}\right)=$ $=\left(4^{n}\right) \cdot\left(\begin{array}{cc}-\frac{1}{2} \frac{(n-1)}{2^{n-1}} & \frac{1}{2} \frac{n}{2^{n}} \\ -2 \frac{n}{2^{n}} & 2 \frac{(n+1)}{2^{n+1}}\end{array}\right)=\left(4^{n}\right) \cdot\left(\begin{array}{cc}-\frac{1}{2} O_{n-1} & \frac{1}{2} O_{n} \\ -2 O_{n} & 2 O_{n+1}\end{array}\right)=\left(\begin{array}{cc}2 O_{n+1} & -\frac{1}{2} O_{n} \\ 2 O_{n} & -\frac{1}{2} O_{n-1}\end{array}\right)^{-1}=\left[M^{n}\right]^{-1}=M^{-n}$.

Cabe observar, por exemplo, que deduzimos de forma semelhante que $M_{k}(-n)=\left(M_{k}(n)\right)^{-1}$. 
No caso em que considerarmos os k-números de Oresme, vamos assumir por indução que $M_{k}(n)=\left(\begin{array}{cc}1 & -\frac{1}{k^{2}} \\ 1 & 0\end{array}\right)^{n}=\left(\begin{array}{cc}2 O_{k, n+1} & -\frac{1}{2} O_{k, n} \\ 2 O_{k, n} & -\frac{1}{2} O_{k, n-1}\end{array}\right)$. Repetindo os argumentos anteriores, poderemos determinar que temos $M_{k}^{n+1}=M_{k}^{n} M_{k}=\left(\begin{array}{cc}1 & -\frac{1}{k^{2}} \\ 1 & 0\end{array}\right)^{n}\left(\begin{array}{cc}1 & -\frac{1}{k^{2}} \\ 1 & 0\end{array}\right)=\left(\begin{array}{cc}k O_{k, n+1} & -\frac{1}{k} O_{k, n} \\ k O_{k, n} & -\frac{1}{k} O_{k, n-1}\end{array}\right)\left(\begin{array}{cc}1 & -\frac{1}{k^{2}} \\ 1 & 0\end{array}\right)=$ $=\left(\begin{array}{cc}k O_{k, n+1}-\frac{1}{k} O_{k, n} & -\frac{1}{k} O_{k, n+1} \\ k O_{k, n}-\frac{1}{k} O_{k, n-1} & -\frac{1}{k} O_{k, n}\end{array}\right)=\left(\begin{array}{cc}k \cdot\left(O_{k, n+1}-\frac{1}{k^{2}} O_{k, n}\right) & -\frac{1}{k} O_{k, n+1} \\ k \cdot\left(O_{k, n}-\frac{1}{k^{2}} O_{k, n-1}\right) & -\frac{1}{k} O_{k, n}\end{array}\right)=\left(\begin{array}{cc}k \cdot O_{k, n+2} & -\frac{1}{k} O_{k, n+1} \\ k \cdot O_{k, n+1} & -\frac{1}{k} O_{k, n}\end{array}\right)=M_{k}(n+1)$.

Para finalizar a verificação dos itens restantes, vejamos a igualdade matricial: $M(m+n)=$ $\left(\begin{array}{cc}2 O_{m+n+1} & -\frac{1}{2} O_{m+n} \\ 2 O_{m+n} & -\frac{1}{2} O_{m+n-1}\end{array}\right)=M^{m+n}=\left(\begin{array}{cc}1 & -\frac{1}{4} \\ 1 & 0\end{array}\right)^{m+n}=\left(\begin{array}{cc}1 & -\frac{1}{4} \\ 1 & 0\end{array}\right)^{m}\left(\begin{array}{cc}1 & -\frac{1}{4} \\ 1 & 0\end{array}\right)^{n}=\left(\begin{array}{cc}2 O_{m+1} & -\frac{1}{2} O_{m} \\ 2 O_{m} & -\frac{1}{2} O_{m-1}\end{array}\right)\left(\begin{array}{cc}2 O_{n+1} & -\frac{1}{2} O_{n} \\ 2 O_{n} & -\frac{1}{2} O_{n-1}\end{array}\right)=$
$=\left(\begin{array}{ll}2 O_{m+1} 2 O_{n+1}-O_{m} O_{n} & -O_{m+1} O_{n}+\frac{1}{4} O_{m} O_{n-1} \\ 2 O_{m} 2 O_{n+1}-O_{m-1} O_{n} & -O_{m} O_{n}+\frac{1}{4} O_{m-1} O_{n-1}\end{array}\right)=\left(\begin{array}{ll}4 O_{m+1} O_{n+1}-O_{m} O_{n} & -O_{m+1} O_{n}+\frac{1}{4} O_{m} O_{n-1} \\ 4 O_{m} O_{n+1}-O_{m-1} O_{n} & -O_{m} O_{n}+\frac{1}{4} O_{m-1} O_{n-1}\end{array}\right)$. Mas, comparando os temos na posição indicada $(1 \times 1)$ podemos determinar a identidade seguinte $O_{m+n}=2 O_{m} \cdot O_{n+1}-\frac{1}{2} O_{m-1} \cdot O_{n}$, para quaisquer inteiros positivos $m, n \geq 0$. Em seguida, a partir do item (ii) escrevemos $M(-(n+m))=M^{-(n+m)}=M^{-n} \cdot M^{-m}=M(-n) \cdot M(-m)$ e basta repetir o argumento anterior afim de encontrar a seguinte identidade $O_{-(m+n)}=2 O_{-m} \cdot O_{-n+1}-\frac{1}{2} O_{-m-1} \cdot O_{-n}$, para quaisquer inteiros positivos $m, n \geq 0$. Para o caso da matriz definida por nós há pouco $M_{k}(n)=\left(\begin{array}{cc}k \cdot O_{k, n+1} & -\frac{1}{k} O_{k, n} \\ k \cdot O_{k, n} & -\frac{1}{k} O_{k, n-1}\end{array}\right)$ repetimos exatamente os mesmos argumentos.

Vejamos, em seguida, a generalização de alguns resultados indicados e/ou desconsiderados por Cerda-Morales (2019).

Teorema 2: Para todo inteiro $m>n \geq 0$ e $k \geq 2$ temos que: (i) $\lim _{n \rightarrow \infty} \frac{O_{n+1}}{O_{n}}=2$; (ii) $\lim _{n \rightarrow \infty} \frac{O_{m}}{O_{n}}=2^{p}$ ; (iii) $\lim _{n \rightarrow \infty} \frac{O_{k, m}}{O_{k, n}}=k^{p}$, no qual $m=n+p$. 
Prova. Para todo inteiro $m \geq n \geq 0$, vamos tomar $p \geq 1$ de sorte que tenhamos a igualdade $m=n+p$. Agora, podemos escrever que $O_{m}=\frac{m}{2^{m}}=\frac{n+p}{2^{n+p}}, O_{-m}=-m \cdot 2^{m}=-(n+p) \cdot 2^{n+p}$. Segue o quociente $\lim _{n \rightarrow \infty} \frac{O_{m}}{O_{n}}=\lim _{n \rightarrow \infty} \frac{O_{n+p}}{O_{n}}=\lim _{n \rightarrow \infty}\left(\frac{\frac{n+p}{2^{n+p}}}{\frac{n}{2^{n}}}\right)=\lim _{n \rightarrow \infty}\left(\frac{n+p}{2^{n+p}}\right) \cdot\left(\frac{2^{n}}{n}\right)=\lim _{n \rightarrow \infty} \frac{n+p}{n} \cdot \frac{2^{n}}{2^{n+p}}=$ $=\lim _{n \rightarrow \infty}\left(1+\frac{p}{n}\right) \cdot \frac{1}{2^{p}}=1 \cdot 2^{p}$, para $p \geq 1$. Para o primeiro item, basta fazer $m=n+1$. No caso quando lidamos especificamente com os k-números de Oresme, podemos verificar que $\lim _{n \rightarrow \infty} \frac{O_{k, m}}{O_{k, n}}=\lim _{n \rightarrow \infty} \frac{O_{k, n+p}}{O_{k, n}}=\lim _{n \rightarrow \infty}\left(\frac{\frac{n+p}{k^{n+p}}}{\frac{n}{k^{n}}}\right)=\lim _{n \rightarrow \infty}\left(\frac{n+p}{k^{n+p}}\right) \cdot\left(\frac{k^{n}}{n}\right)=\lim _{n \rightarrow \infty}\left(1+\frac{p}{n}\right) \cdot\left(\frac{k^{n}}{k^{n+p}}\right)=k^{p}$.

No teorema seguinte, acrescentaremos alguns resultados desconsiderados por Horadam (1974), entretanto, Horadam (1974) exibe apenas a função geradora $g(x)$.

Teorema 3: A função geradora correspondente aos números de Oresme e aos k-números de Oresme são, respectivamente, dadas por $g(x)=\frac{\frac{1}{2} x}{1-x+\frac{1}{4} x^{2}}$ e $g_{k}(x)=\frac{\frac{1}{k} x}{1-x+\frac{1}{k^{2}} x^{2}}$.

Prova. De imediato, vamos recordar as seguintes relações fundamentais $O_{n+2}-O_{n+1}+\frac{1}{4} O_{n}=0$ e $O_{k, n+2}-O_{k, n+1}+\frac{1}{k^{2}} O_{k, n}=0$. Consideraremos as seguintes somas infinitas formais indicadas $g(x)=\sum_{i=0}^{\infty} O_{i} x^{i}$ e $g_{k}(x)=\sum_{i=0}^{\infty} O_{k, i} x^{i}$ cujos coeficientes são, exatamente, os números de Oresme e aos k-números de Oresme. Em seguida, vamos descrever as relações abaixo:

$$
\left\{\begin{array}{l}
g(x)=O_{0}+O_{1} x+O_{2} x^{2}+O_{3} x^{3}+O_{4} x^{4}+O_{5} x^{5} \cdots+O_{n} x^{n}+ \\
-x \cdot g(x)=-O_{0} x-O_{1} x^{2}-O_{2} x^{3}-\cdots-O_{n-1} x^{n}-O_{n} x^{n+1}-O_{n+1} x^{n+2}-\cdots \\
\frac{1}{4} x^{2} \cdot g(x)=\frac{1}{4} O_{0} x^{2}+\frac{1}{4} O_{1} x^{3}+\frac{1}{4} O_{2} x^{4}+\cdots+\frac{1}{4} O_{n-2} x^{n}+\frac{1}{4} O_{n-1} x^{n+1}+\cdots
\end{array}\right.
$$

No passo seguinte, vamos tomar a expressão $\left(g(x)-x \cdot g(x)+\frac{1}{4} x^{2} \cdot g(x)\right)=O_{0}+\left(O_{1}-O_{0}\right) x+$ $\left(O_{2}-O_{1}+\frac{1}{4} O_{0}\right) x^{2}+\left(O_{3}-O_{2}+\frac{1}{4} O_{1}\right) x^{2}+\cdots+\left(O_{n+2}-O_{n+1}+\frac{1}{4} O_{n}\right) x^{2}+\cdots=0+\frac{1}{2} x+0 x^{2}+0 x^{3}+\cdots$ $+0 x^{n}+\cdots$. Finalmente, determinaremos que $\left(1-x+\frac{1}{4} x^{2}\right) \cdot g(x)=\frac{1}{2} x$. No segundo caso, repetimos os produtos necessários e que devem resultar, para o caso dos k-números de Oresme, na relação que segue $g_{k}(x)-x \cdot g_{k}(x)+\frac{1}{k^{2}} x^{2} \cdot g_{k}(x)=O_{k, 0}+\left(O_{k, 1}-O_{k, 0}\right) x$ 
Na seção subsequente introduziremos algumas propriedades sobre os polinômios de Oresme.

\section{Os polinômios de Oresme}

A partir do trabalho de Cerda-Morales (2019) consideraremos a seguinte definição.

Definição 3: Dada uma variável não nula os números do tipo $\left\{O_{n}(x)\right\}_{n \in I N}$ são definidos pela relação homogênea de recorrência $O_{n+1}(x)=\left\{\begin{array}{l}\frac{1}{x}, n=0,1 \\ O_{n}(x)-\frac{1}{x^{2}} O_{n-1}(x), n \geq 2\end{array}\right.$.

Com origem nessa definição, Cerda-Morales (2019) exibe alguns de seus elementos iniciais: $O_{0}(x)=0, O_{1}(x)=\frac{1}{x}, O_{2}(x)=\frac{1}{x}, O_{3}(x)=\frac{x^{2}-1}{x^{3}}, O_{4}(x)=\frac{x^{2}-2}{x^{3}}, O_{5}(x)=\frac{x^{4}-3 x^{2}+1}{x^{5}}, O_{6}(x)=\frac{x^{4}-4 x^{2}+3}{x^{5}}$.

De forma semelhante ao pensamento de Horadam (1974) podemos determinar os elementos do seguinte conjunto $\left\{O_{-n}(x)\right\}_{n \in I N}$. De fato, vejamos que $O_{1}(x)=O_{0}(x)-\frac{1}{x^{2}} O_{-1}(x) \leftrightarrow O_{-1}(x)=0$. No caso seguinte, vejamos que $O_{0}(x)=O_{-1}(x)-\frac{1}{x^{2}} O_{-2}(x) \leftrightarrow O_{-2}(x)=-x$ e segue os mesmos argumentos que permitem determinar que temos: $O_{-3}(x)=-x^{3}, O_{-4}(x)=-x^{3}\left(x^{2}-1\right), O_{-5}(x)=$ $=-x^{5}\left(x^{2}-2\right), O_{-6}(x)=-x^{5}\left(x^{4}-3 x^{2}+1\right), O_{-7}(x)=-x^{11}+4 x^{9}-3 x^{7}, O_{-8}(x)=-x^{13}+5 x^{11}-6 x^{9}+x^{7}$, $O_{-9}(x)=-x^{15}+6 x^{13}-10 x^{11}+4 x^{9}, O_{-10}(x)=-x^{15}+6 x^{13}-10 x^{11}+4 x^{9}$, etc.

Cerda-Morales (2019) considera o procedimento de resolução da relação de recorrência $O_{n+1}(x)=O_{n}(x)-\frac{1}{x^{2}} O_{n-1}(x)$ afim de determinar a equação característica. De fato, basta ver que $\frac{O_{n+1}(x)}{O_{n}(x)}=1-\frac{1}{x^{2}} \frac{O_{n-1}(x)}{O_{n}(x)}=1-\frac{1}{x^{2}} \frac{1}{\left(\frac{O_{n}(x)}{O_{n-1}(x)}\right)}$. No passo seguinte, devemos determinar a seguinte equação $x^{2} \cdot t^{2}-x^{2} \cdot t+1=0$ ou $t^{2}-t+\frac{1}{x^{2}}=0$, cujas raízes indicadas por Cerda-Morales (2019) são dadas pelas expressões e propriedades $\lambda_{1}(x)=\frac{x+\sqrt{x^{2}-4}}{2 x}, \lambda_{2}(x)=\frac{x-\sqrt{x^{2}-4}}{2 x}$, $\lambda_{1}(x) \cdot \lambda_{2}(x)=\frac{1}{x^{2}}, \frac{1}{\lambda_{1}(x)}=x^{2} \cdot \lambda_{2}(x), \lambda_{1}(x)+\lambda_{2}(x)=-1$. Em seguida, vejamos o seguinte teorema que corresponde a determinação da fórmula de Binet para os polinômios de Oresme e que acrescenta uma correção e generalização ao resultado exibido por Cerda-Morales (2019).

Teorema 4: Para todo inteiro positivo $n \geq 0$, temos as seguintes fórmulas de Binet: (i) $O_{n}(x)=\frac{\lambda_{1}^{n}(x)-\lambda_{2}^{n}(x)}{x \sqrt{x^{2}-4}}$; (ii) $O_{-n}(x)=(-1) \cdot x^{2 n} \cdot\left(\frac{\lambda_{1}(x)^{n}-\lambda_{2}(x)^{n}}{x \sqrt{x^{2}-4}}\right)=(-1) \cdot x^{2 n} \cdot O_{n}(x)$. 
Prova. Vamos considerar a equação $t^{2}-t+\frac{1}{x^{2}}=0$ cujas raízes indicadas por Cerda-Morales (2019) são dadas por $\lambda_{1}(x)=\frac{x+\sqrt{x^{2}-4}}{2 x}, \lambda_{2}(x)=\frac{x-\sqrt{x^{2}-4}}{2 x}$. Reparemos que valem as relações $\lambda_{1}(x)^{2}=\lambda_{1}(x)-\frac{1}{x^{2}}$ e $\lambda_{2}(x)^{2}=\lambda_{2}(x)-\frac{1}{x^{2}}=x \cdot\left(\frac{1}{x}\right) \cdot \lambda_{2}(x)-\frac{1}{x}\left(\frac{1}{x}\right)=x \cdot O_{2}(x) \lambda_{2}(x)-\frac{1}{x} \cdot O_{1}(x)$.

Vamos observar que $\lambda_{1}(x)^{3}=\lambda_{1}(x)\left(\lambda_{1}(x)-\frac{1}{x^{2}}\right)=\lambda_{1}(x)^{2}-\frac{1}{x^{2}} \lambda_{1}(x)=\lambda_{1}(x)-\frac{1}{x^{2}}-\frac{1}{x^{2}} \lambda_{1}(x)$ $=\lambda_{1}(x)\left(\frac{x^{2}-1}{x^{2}}\right)-\frac{1}{x^{2}}=x \cdot \lambda_{1}(x)\left(\frac{x^{2}-1}{x^{3}}\right)-\frac{1}{x}\left(\frac{1}{x}\right)=x \cdot \lambda_{1}(x) O_{3}(x)-\frac{1}{x} O_{2}(x)$. Em seguida, podemos verificar que $\lambda_{1}(x)^{4}=\lambda_{1}(x)^{2} \lambda_{1}(x)^{2}=\left(\lambda_{1}(x)-\frac{1}{x^{2}}\right)\left(\lambda_{1}(x)-\frac{1}{x^{2}}\right)=\lambda_{1}(x)^{2}-2 \frac{1}{x^{2}} \lambda_{1}(x)+\frac{1}{x^{4}}$ $=\lambda_{1}(x)-\frac{1}{x^{2}}-2 \cdot \frac{1}{x^{2}} \cdot \lambda_{1}(x)+\frac{1}{x^{4}}=\lambda_{1}(x) \cdot\left(\frac{x^{2}-2}{x^{2}}\right)+\frac{1}{x^{4}}-\frac{1}{x^{2}}=x \cdot \lambda_{1}(x)\left(\frac{x^{2}-2}{x^{3}}\right)-\left(\frac{x^{2}-1}{x^{4}}\right)=$ $=x \cdot \lambda_{1}(x)\left(\frac{x^{2}-2}{x^{3}}\right)-\frac{1}{x}\left(\frac{x^{2}-1}{x^{3}}\right)=x \cdot \lambda_{1}(x) O_{4}(x)-\frac{1}{x} \cdot O_{3}(x)$. Assumiremos por indução que $\lambda_{1}(x)^{n}=x \cdot O_{n}(x) \lambda_{1}(x)-\frac{1}{x} \cdot O_{n-1}(x)$. Assim, basta verificar que $\lambda_{1}(x)^{n+1}=\lambda_{1}(x)^{n} \lambda_{1}(x)=$ $=\lambda_{1}(x) \cdot\left(x \cdot O_{n}(x) \lambda_{1}(x)-\frac{1}{x} \cdot O_{n-1}(x)\right)=x \cdot O_{n}(x) \cdot \lambda_{1}(x) \lambda_{1}(x)-\frac{1}{x^{2}} \cdot x \cdot O_{n-1}(x) \cdot \lambda_{1}(x)=$ $=x \cdot O_{n}(x)\left(\lambda_{1}(x)-\frac{1}{x^{2}}\right)-x \frac{1}{x^{2}} \cdot O_{n-1}(x) \lambda_{1}(x)=x \cdot \lambda_{1}(x) \cdot\left(O_{n}(x)-\frac{1}{x^{2}} \cdot O_{n-1}(x)\right)-\frac{1}{x} \cdot O_{n}(x)$ $=x \cdot \lambda_{1}(x) \cdot O_{n+1}(x)-\frac{1}{x} \cdot O_{n}(x)$. Por conseguinte, podemos considerar o seguinte sistema $\left\{\begin{array}{l}\lambda_{1}(x)^{n}=x \cdot O_{n}(x) \lambda_{1}(x)-\frac{1}{x} \cdot O_{n-1}(x) \\ \lambda_{2}(x)^{n}=x \cdot O_{n}(x) \lambda_{2}(x)-\frac{1}{x} \cdot O_{n-1}(x)\end{array}\right.$ e, tomando a seguinte diferença $\left(\lambda_{1}(x)^{n}-\lambda_{2}(x)^{n}\right)=$ $=x \cdot O_{n}(x) \lambda_{1}(x)-\frac{1}{x} \cdot O_{n-1}(x)-x \cdot O_{n}(x) \lambda_{2}(x)+\frac{1}{x} \cdot O_{n-1}(x)=x \cdot O_{n}(x) \cdot\left(\lambda_{1}(x)-\lambda_{2}(x)\right)$. Finalmente, determinamos a fórmula de Binet indicada por $O_{n}(x)=\frac{1}{x} \frac{\lambda_{1}(x)^{n}-\lambda_{2}(x)^{n}}{\lambda_{1}(x)-\lambda_{2}(x)}$. Por outro lado, de forma e interesse semelhante ao que indicamos inicialmente, buscamos determinar o comportamento dos polinômios de Mersenne com índices inteiros negativos. Para tanto, vamos substituir na fórmula anterior os índices $(n)$ por $(-n)$ afim de determinar os elementos do conjunto $\left\langle O_{-n}(x)\right\rangle_{n \in I N}$. Basta ver ocorre $O_{-n}(x)=\frac{1}{x} \frac{\lambda_{1}^{-n}(x)-\lambda_{2}^{-n}(x)}{\sqrt{x^{2}-4}}=\frac{1}{x} \frac{\left(\frac{1}{\lambda_{1}(x)}\right)^{n}-\left(\frac{1}{\lambda_{2}(x)}\right)^{n}}{\sqrt{x^{2}-4}}$ 
$=\frac{\left(x^{2} \lambda_{2}(x)\right)^{n}-\left(x^{2} \lambda_{1}(x)\right)^{n}}{x \sqrt{x^{2}-4}}=-x^{2 n}\left(\frac{\lambda_{1}(x)^{n}-\lambda_{2}(x)^{n}}{x \sqrt{x^{2}-4}}\right)=(-1) x^{2 n} \cdot\left(\frac{\lambda_{1}(x)^{n}-\lambda_{2}(x)^{n}}{x \sqrt{x^{2}-4}}\right)=(-1) \cdot x^{2 n} \cdot O_{n}(x)$.

Em seguida, vejamos o comportamento das potências das seguintes matrizes $M^{n}(x)$ :

$$
\begin{aligned}
& M(x)=\left(\begin{array}{cc}
1 & -\frac{1}{x^{2}} \\
1 & 0
\end{array}\right)=\left(\begin{array}{rr}
x \cdot \frac{1}{x} & -\frac{1}{x} \cdot \frac{1}{x} \\
x \cdot \frac{1}{x} & -\frac{1}{x} \cdot 0
\end{array}\right)=\left(\begin{array}{cc}
x \cdot O_{2}(x) & -\frac{1}{x} \cdot O_{1}(x) \\
x \cdot O_{1}(x) & -\frac{1}{x} \cdot O_{0}(x)
\end{array}\right), M^{2}(x)=\left(\begin{array}{cc}
\frac{x^{2}-1}{x^{2}} & -\frac{1}{x^{2}} \\
1 & -\frac{1}{x^{2}}
\end{array}\right) \\
& \left(\begin{array}{cc}
x \cdot\left(\frac{x^{2}-1}{x^{3}}\right) & -\frac{1}{x} \cdot \frac{1}{x} \\
x \cdot \frac{1}{x} & -\frac{1}{x} \cdot \frac{1}{x}
\end{array}\right)=\left(\begin{array}{cc}
x \cdot O_{3}(x) & -\frac{1}{x} \cdot O_{2}(x) \\
x \cdot O_{2}(x) & -\frac{1}{x} \cdot O_{1}(x)
\end{array}\right), M^{3}(x)=\left(\begin{array}{cc}
\frac{x^{2}-2}{x^{2}} & -\frac{x^{2}-1}{x^{4}} \\
\frac{x^{2}-1}{x^{2}} & -\frac{1}{x^{2}}
\end{array}\right)=, \\
& =\left(\begin{array}{cc}
x \cdot\left(\frac{x^{2}-2}{x^{3}}\right) & -\frac{1}{x} \cdot\left(\frac{x^{2}-1}{x^{3}}\right) \\
x \cdot\left(\frac{x^{2}-1}{x^{3}}\right) & -\frac{1}{x} \cdot\left(\frac{1}{x}\right)
\end{array}\right)=\left(\begin{array}{cc}
x \cdot O_{4}(x) & -\frac{1}{x} \cdot O_{3}(x) \\
x \cdot O_{3}(x) & -\frac{1}{x} \cdot O_{2}(x)
\end{array}\right), M^{4}(x)=\left(\begin{array}{cc}
\frac{x^{4}-3 x^{2}+1}{x^{4}} & -\frac{x^{2}-2}{x^{4}} \\
\frac{x^{2}-2}{x^{2}} & -\frac{x^{2}-1}{x^{4}}
\end{array}\right)= \\
& =\left(\begin{array}{cc}
x \cdot\left(\frac{x^{4}-3 x^{2}+1}{x^{5}}\right) & -\frac{1}{x} \cdot\left(\frac{x^{2}-2}{x^{3}}\right) \\
x \cdot\left(\frac{x^{2}-2}{x^{3}}\right) & -\frac{1}{x} \cdot\left(\frac{x^{2}-1}{x^{3}}\right)
\end{array}\right)=\left(\begin{array}{cc}
x \cdot O_{5}(x) & -\frac{1}{x} \cdot O_{4}(x) \\
x \cdot O_{4}(x) & -\frac{1}{x} \cdot O_{3}(x)
\end{array}\right) . \text { Logo em seguida, vejamos o }
\end{aligned}
$$

comportamento correspondente das matrizes inversas que indicamos por: $M(x)^{-1}=\left(\begin{array}{cc}0 & 1 \\ -x^{2} & x^{2}\end{array}\right)=$ $\left(\begin{array}{cc}x \cdot & -\frac{1}{x}(-x) \\ x \cdot(-x) & -\frac{1}{x}\left(-x^{3}\right)\end{array}\right)=\left(\begin{array}{cc}x \cdot O_{0}(x) & -\frac{1}{x} \cdot O_{-1}(x) \\ x \cdot O_{-1}(x) & -\frac{1}{x} \cdot O_{-2}(x)\end{array}\right), M(x)^{-2}=\left(\begin{array}{cc}-x^{2} & x^{2} \\ -x^{4} & x^{4}-x^{2}\end{array}\right)=\left(\begin{array}{cc}x \cdot(-x) & -\frac{1}{x}\left(-x^{3}\right) \\ x \cdot\left(-x^{3}\right) & x^{4}-x^{2}\end{array}\right)$
$=\left(\begin{array}{cc}x \cdot O_{-1}(x) & -\frac{1}{x} \cdot O_{-2}(x) \\ x \cdot O_{-n}(x) & -\frac{1}{x} \cdot O_{-3}(x)\end{array}\right), M(x)^{-3}=\left(\begin{array}{cc}-x^{4} & x^{4}-x^{2} \\ -x^{6}+x^{4} & x^{6}-2 x^{4}\end{array}\right)=\left(\begin{array}{cc}x \cdot O_{-2}(x) & -\frac{1}{x} \cdot O_{-3}(x) \\ x \cdot O_{-3}(x) & -\frac{1}{x} \cdot O_{-4}(x)\end{array}\right)$, etc.

Vamos definir, a partir desses exemplos particulares, as seguintes matrizes indicadas por $M_{n}(x)=\left(\begin{array}{cc}x \cdot O_{n+1}(x) & -\frac{1}{x} \cdot O_{n}(x) \\ x \cdot O_{n}(x) & -\frac{1}{x} \cdot O_{n-1}(x)\end{array}\right)$ e $M_{-n}(x)=\left(\begin{array}{cc}x \cdot O_{-n+1}(x) & -\frac{1}{x} \cdot O_{-n}(x) \\ x \cdot O_{-n}(x) & -\frac{1}{x} \cdot O_{-n-1}(x)\end{array}\right)$ são introduzidas. Com origem nesses casos particulares e as matrizes $M_{n}(x), M_{-n}(x)$, com $n \geq 0$ agora definidas, enunciaremos o seguinte teorema.

Teorema 5: Para todo inteiro positivo $n \geq 0$ valem as seguintes relações:

ALVES, F. R. V. Sequência de Oresme e algumas propriedades (matriciais) generalizadas. C.Q.D.- Revista Eletrônica Paulista de Matemática, Bauru, v. 16, p. 28-52, dez. 2019 .

DOI: 10.21167/cqdvol16201923169664frva2852 Disponível em: http://www.fc.unesp.br/\#!/departamentos/matematica/revista-cqd/ 
(i) $M_{n}(x)=M(x)^{n}=\left(\begin{array}{cc}x \cdot O_{n+1}(x) & -\frac{1}{x} \cdot O_{n}(x) \\ x \cdot O_{n}(x) & -\frac{1}{x} \cdot O_{n-1}(x)\end{array}\right)$; (ii) $O_{n-1}(x) \cdot O_{n+1}(x)-O_{n}(x)^{2}=-\frac{1}{x^{2 n}}$;

(iii) $M_{-n}(x)=(-1) \cdot x^{2 n}\left(M_{n}(x)\right)^{-1}=(-1) \cdot x^{2 n}\left(M(x)^{n}\right)^{-1}=(-1) \cdot x^{2 n} \cdot M(x)^{n}$.

Prova. Os casos particulares podem ser apreciados nos trechos predecessores. No passo de indução matemática, vamos admitir que $M_{n}(x)=M(x)^{n}=\left(\begin{array}{cc}x \cdot O_{n+1}(x) & -\frac{1}{x} \cdot O_{n}(x) \\ x \cdot O_{n}(x) & -\frac{1}{x} \cdot O_{n-1}(x)\end{array}\right)$, para todo inteiro positivo e, em seguida, observar o comportamento da matriz da potência $M(x)^{n+1}=$

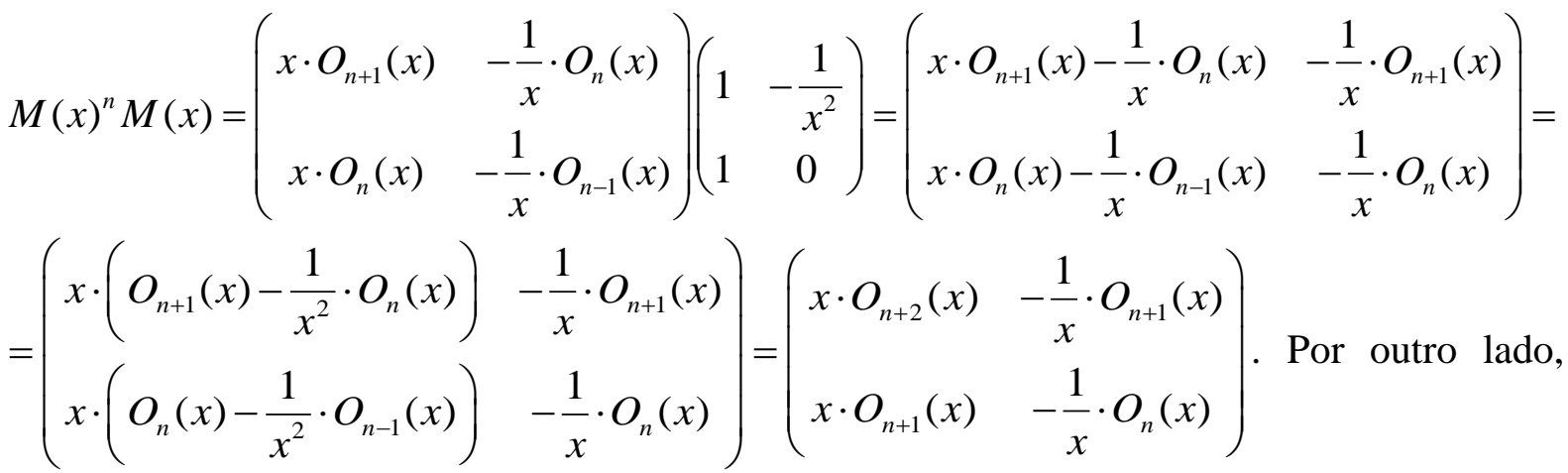
para o segundo item, vamos considerar a seguinte matriz inversa indicada por: $\left(M_{n}(x)\right)^{-1}=$ $\left(\begin{array}{cc}x \cdot O_{n+1}(x) & -\frac{1}{x} \cdot O_{n}(x) \\ x \cdot O_{n}(x) & -\frac{1}{x} \cdot O_{n-1}(x)\end{array}\right)^{-1}=\left(\begin{array}{cc}-\frac{1}{x} \cdot O_{n-1}(x) & -x \cdot O_{n}(x) \\ \frac{1}{x} \cdot O_{n}(x) & x \cdot O_{n+1}(x)\end{array}\right)^{T}=\left(\begin{array}{cc}-\frac{1}{x} \cdot O_{n-1}(x) & \frac{1}{x} \cdot O_{n}(x) \\ -x \cdot O_{n}(x) & x \cdot O_{n+1}(x)\end{array}\right)$. Por outro lado, substituindo os índices $(n)$ por $(-n)$, vejamos que se tomarmos a matriz $M_{-n}(x)=$ $\left(\begin{array}{cc}x \cdot O_{-n+1}(x) & -\frac{1}{x} \cdot O_{-n}(x) \\ x \cdot O_{-n}(x) & -\frac{1}{x} \cdot O_{-n-1}(x)\end{array}\right)=\left(\begin{array}{cc}x \cdot O_{-(n-1)}(x) & -\frac{1}{x} \cdot O_{-n}(x) \\ x \cdot O_{-n}(x) & -\frac{1}{x} \cdot O_{-(n+1)}(x)\end{array}\right)=\left(\begin{array}{cc}-x \cdot x^{2(n-1)} \cdot O_{n-1}(x) & \frac{1}{x} \cdot x^{2 n} \cdot O_{n}(x) \\ -x \cdot x^{2 n} \cdot O_{n}(x) & \frac{1}{x} \cdot x^{2(n+1)} \cdot O_{n+1}(x)\end{array}\right)$ $=\left(\begin{array}{cc}-x \cdot x^{2 n} \cdot x^{-2} \cdot O_{n-1}(x) & \frac{1}{x} \cdot x^{2 n} \cdot O_{n}(x) \\ -x \cdot x^{2 n} \cdot O_{n}(x) & \frac{1}{x} \cdot x^{2 n} \cdot x^{2} \cdot O_{n+1}(x)\end{array}\right)=(-1) \cdot x^{2 n} \cdot\left(\begin{array}{cc}-\frac{1}{x} \cdot O_{n-1}(x) & \frac{1}{x} \cdot O_{n}(x) \\ -x \cdot O_{n}(x) & x \cdot O_{n+1}(x)\end{array}\right)=(-1) \cdot x^{2 n}\left(M_{n}(x)\right)^{-1}$.

Segue, pois, a seguinte relação matricial $M_{-n}(x)=(-1) \cdot x^{2 n}\left(M_{n}(x)\right)^{-1}, n \geq 1$.

Vejamos, em seguida, propriedades semelhantes ao que já observamos anteriormente no caso dos k-números de Oresme aplicadas ao estudo dos polinômios de Oresme.

Teorema 6: Para quaisquer inteiros positivos $m, n \geq 0$ valem as relações. 
(i) $M_{m+n}(x)=M(x)^{n+m}=\left(\begin{array}{cc}x \cdot O_{n+m+1}(x) & -\frac{1}{x} \cdot O_{n+m}(x) \\ x \cdot O_{n+m}(x) & -\frac{1}{x} \cdot O_{n+m-1}(x)\end{array}\right), O_{n+m}(x)=x \cdot O_{n}(x) \cdot O_{m+1}(x)-\frac{1}{x} O_{n-1}(x) \cdot O_{m}(x)$;

(ii) $M_{-(m+n)}(x)=M(x)^{-(n+m)}=\left(\begin{array}{cc}x \cdot O_{-n-m+1}(x) & -\frac{1}{x} \cdot O_{-n-m}(x) \\ x \cdot O_{-n-m}(x) & -\frac{1}{x} \cdot O_{-n-m-1}(x)\end{array}\right), O_{-(n+m)}(x)=x \cdot O_{-n}(x) \cdot O_{-m+1}(x)-\frac{1}{x} O_{-n-1}(x) \cdot O_{-m}(x)$.

Prova. De imediato, basta usar a igualdade mediante a definição $M_{m+n}(x)=$ $\left(\begin{array}{cc}x \cdot O_{n+m+1}(x) & -\frac{1}{x} \cdot O_{n+m}(x) \\ x \cdot O_{n+m}(x) & -\frac{1}{x} \cdot O_{n+m-1}(x)\end{array}\right)=M(x)^{n} \cdot M(x)^{m}=\left(\begin{array}{cc}x \cdot O_{n+1}(x) & -\frac{1}{x} \cdot O_{n}(x) \\ x \cdot O_{n}(x) & -\frac{1}{x} \cdot O_{n-1}(x)\end{array}\right)\left(\begin{array}{cc}x \cdot O_{m+1}(x) & -\frac{1}{x} \cdot O_{m}(x) \\ x \cdot O_{m}(x) & -\frac{1}{x} \cdot O_{m-1}(x)\end{array}\right)$ $=\left(\begin{array}{ll}x^{2} \cdot O_{n+1}(x) \cdot O_{m+1}(x)-O_{n}(x) \cdot O_{m}(x) & -O_{n+1}(x) O_{m}(x)+\frac{1}{x^{2}} \cdot O_{n}(x) \cdot O_{m-1}(x) \\ x^{2} \cdot O_{n}(x) \cdot O_{m+1}(x)-O_{n-1}(x) \cdot O_{m}(x) & -O_{n}(x) \cdot O_{m}(x)+\frac{1}{x^{2}} \cdot O_{n-1}(x) O_{m-1}(x)\end{array}\right)$. Finalmente, comparando os termos na posição $(1 \times 1)$ deduzimos a seguinte igualdade $O_{n+m}(x)=x \cdot O_{n}(x) \cdot O_{m+1}(x)-\frac{1}{x} O_{n-1}(x) \cdot O_{m}(x)$, para quaisquer inteiros positivos $m, n \geq 0$. Os mesmos argumentos são repetidos para o segundo item e seguem os resultados .

\section{Os Quaternions de Oresme}

A partir da literatura especializada, constatamos o interesse de representações por intermédio dos Quaterniôns para algumas sequências recorrentes, todavia, não registramos resultados correspondentes aos números de Oresme. Assim, com origem em determinados argumentos indicados nas seções predecessoras, vamos introduzir a seguinte definição que corresponde a uma nova noção introduzida no presente trabalho.

Definição 4: Chamamos de Quaternion de Oresme de ordem 'n' ao elemento descrito por $Q O_{n}=O_{n}+O_{n+1} \vec{i}+O_{n+2} \vec{j}+O_{n+3} \vec{k}$, em que os coeficientes são os números de Oresme e as unidades imaginárias são descritas pelas operações formais $\vec{i}^{2}=\vec{j}^{2}=\vec{k}^{2}=-1, \vec{i} \cdot \vec{j}=\vec{k}=-\vec{j} \cdot \vec{i}$.

Com origem na definição, determinamos: $Q O_{-1}=O_{-1}+O_{0} i+O_{1} j+O_{2} k=-2+0 i+\frac{1}{2} j+\frac{2}{4} k$. $Q O_{0}=0+\frac{1}{2} i+\frac{2}{4} j+\frac{3}{8} k, Q O_{1}=\frac{1}{2}+\frac{2}{4} i+\frac{3}{8} j+\frac{4}{16} k, Q O_{2}=\frac{2}{4}+\frac{3}{8} i+\frac{4}{16} j+\frac{5}{32} k$, etc.

E, logo em seguida, definiremos um k-Quaternion de Oresme.

Definição 5: Para todo inteiro $k \geq 3$, chamamos de k-Quaternion de Oresme de ordem ' $\mathrm{n}$ ' ao elemento descrito por $Q O_{k, n}=O_{k, n}+O_{k, n+1} \vec{i}+O_{k, n+2} \vec{j}+O_{k, n+3} \vec{k}$, em que os coeficientes são 
os números de Oresme e as unidades imaginárias são descritas pelas operações formais $\vec{i}^{2}=\vec{j}^{2}=\vec{k}^{2}=-1, \vec{i} \cdot \vec{j}=\vec{k}=-\vec{j} \cdot \vec{i}$.

Vejamos, pois, alguns dos seus elementos preliminares: $Q O_{k,-1}=-k+0 \vec{i}+\frac{1}{k} \vec{j}+\frac{1}{k} \vec{k}$, $Q O_{k, 0}=0+\frac{1}{k} \vec{i}+\frac{1}{k} \vec{j}+\frac{k^{2}-1}{k^{3}} \vec{k}, Q O_{k, 1}=\frac{1}{k}+\frac{1}{k} \bar{i}+\frac{k^{2}-1}{k^{3}} \vec{j}+\frac{k^{2}-2}{k^{3}} \vec{k}$, etc. Decorrem, imediatamente, as seguintes propriedades relacionadas com os Quaternions de Oresme.

Teorema 7: Valem as seguintes relações fundamentais: (i) $Q O_{n+2}=Q O_{n+1}-\frac{1}{4} Q O_{n}$; (ii) $Q O_{k, n+2}=Q O_{k, n+1}-\frac{1}{4} Q O_{k, n} ; \quad$ (iii) $\quad Q O_{n}=\frac{1}{2^{n}}\left(n+\frac{n+1}{2} \vec{i}+\frac{n+2}{4} \vec{j}+\frac{n+3}{8} \vec{k}\right)$;

$Q O_{-n}=-2^{n}\left(n+\frac{n-1}{2} \vec{i}+\frac{n-2}{4} \vec{j}+\frac{n-3}{8} \vec{k}\right)$; (iv) $Q O_{k, n}=\frac{1}{k^{n}}\left(n+\frac{n+1}{k} \vec{i}+\frac{n+2}{k^{2}} \vec{j}+\frac{n+3}{k^{3}} \vec{k}\right)$.

Prova. No primeiro item, da definição anterior, desenvolvemos o seguinte termo da direita e $\operatorname{vemos}\left(Q O_{n+1}-\frac{1}{4} Q O_{n}\right)=\left(O_{n+1}+O_{n+2} \vec{i}+O_{n+3} \vec{j}+O_{n+4} \vec{k}\right)-\frac{1}{4}\left(O_{n}+O_{n+1} \vec{i}+O_{n+2} \vec{j}+O_{n+3} \vec{k}\right)=O_{n+1}-\frac{1}{4} O_{n}+$ $O_{n+2} \vec{i}-\frac{1}{4} O_{n+1} \vec{i}+O_{n+3} \vec{j}-\frac{1}{4} O_{n+2} \vec{j}+O_{n+4} \vec{k}-\frac{1}{4} O_{n+3} \vec{k}=Q O_{n+2}$. Para o segundo item, repetiremos o mesmo procedimento, de sorte que $Q O_{k,-n+2}=Q O_{k,-n+1}-\frac{1}{4} Q O_{k,-n}$. Por fim, vejamos que temos $Q O_{n}=\frac{1}{2^{n}}\left(n+\frac{n+1}{2} \vec{i}+\frac{n+2}{4} \vec{j}+\frac{n+3}{8} \vec{k}\right)$ e, substituindo os índices correspondentes, encontraremos $Q O_{-n}=\frac{1}{2^{-n}}\left(-n+\frac{-n+1}{2} \vec{i}+\frac{-n+2}{4} \vec{j}+\frac{-n+3}{8} \vec{k}\right)=-2^{n}\left(n+\frac{n-1}{2} \vec{i}+\frac{n-2}{4} \vec{j}+\frac{n-3}{8} \vec{k}\right)$. Vemos ainda que podemos escrever o quaternion $Q O_{k, n}=\frac{n}{k^{n}}+\frac{n+1}{k^{n+1}} \vec{i}+\frac{n+2}{k^{n+2}} \vec{j}+\frac{n+3}{k^{n+3}} \vec{k}=$ $\frac{1}{k^{n}} \cdot\left(n+\frac{n+1}{k} \vec{i}+\frac{n+2}{k^{2}} \vec{j}+\frac{n+3}{k^{3}} \vec{k}\right)$

Considerar as seguintes matrizes escolhidas $M=\left(\begin{array}{cc}1 & -\frac{1}{4} \\ 1 & 0\end{array}\right), M_{Q}=\left(\begin{array}{cc}2 Q_{1} & -\frac{1}{2} Q_{0} \\ 2 Q_{0} & -\frac{1}{2} Q_{-1}\end{array}\right)=$ $=\left(\begin{array}{cc}2\left(\frac{1}{2}+\frac{2}{4} \vec{i}+\frac{3}{8} \vec{j}+\frac{4}{16} \vec{k}\right) & -\frac{1}{2}\left(0+\frac{1}{2} i+\frac{2}{4} j+\frac{3}{8} k\right) \\ 2\left(0+\frac{1}{2} \vec{i}+\frac{2}{4} \vec{j}+\frac{3}{8} \vec{k}\right) & -\frac{1}{2}\left(-2+0 i+\frac{1}{2} j+\frac{2}{4} k\right)\end{array}\right)$ e $M_{Q, k}=\left(\begin{array}{cc}2 Q_{k, 1} & -\frac{1}{2} Q_{k, 0} \\ 2 Q_{k .0} & -\frac{1}{2} Q_{k,-1}\end{array}\right)=$ 


$$
=\left(\begin{array}{cc}
2\left(\frac{1}{k}+\frac{1}{k} \vec{i}+\frac{k^{2}-1}{k^{3}} \vec{j}+\frac{k^{2}-2}{k^{3}} \vec{k}\right) & -\frac{1}{2}\left(0+\frac{1}{k} \vec{i}+\frac{1}{k} \vec{j}+\frac{k^{2}-1}{k^{3}} \vec{k}\right) \\
2\left(0+\frac{1}{k} \vec{i}+\frac{1}{k} \vec{j}+\frac{k^{2}-1}{k^{3}} \vec{k}\right) & -\frac{1}{2}\left(-k+0 \vec{i}+\frac{1}{k} \vec{j}+\frac{1}{k} \vec{k}\right)
\end{array}\right)
$$

Podemos verificar

que ocorrem as seguintes propriedades do produto de matrizes

$$
\begin{aligned}
& M Q_{O}=Q_{O} M=\left(\begin{array}{cc}
1+\frac{3}{4} \vec{i}+\frac{2}{4} \vec{j}+\frac{5}{16} \vec{k} & -\frac{1}{4}-\frac{1}{4} \vec{i}-\frac{3}{16} \vec{j}-\frac{1}{8} \vec{k} \\
1+\vec{i}+\frac{3}{4} \vec{j}+\frac{1}{2} \vec{k} & 0-\frac{1}{4} \vec{i}-\frac{1}{4} \vec{j}-\frac{3}{16} \vec{k}
\end{array}\right)=\left(\begin{array}{cc}
2 Q O_{2} & -\frac{1}{2} Q O_{1} \\
2 Q O_{1} & -\frac{1}{2} Q O_{0}
\end{array}\right), \\
& M^{2} \cdot Q_{O}=\left(\begin{array}{cc}
\frac{3}{4}+\frac{1}{2} \vec{i}+\frac{5}{16} \vec{j}+\frac{3}{16} \vec{k} & -\frac{1}{4}-\frac{3}{16} \vec{i}-\frac{1}{8} \vec{j}-\frac{5}{64} \vec{k} \\
1+\frac{3}{4} \vec{i}+\frac{1}{2} \vec{j}+\frac{5}{16} \vec{k} & -\frac{1}{4}-\frac{1}{4} \vec{i}-\frac{3}{16} \vec{j}-\frac{1}{8} \vec{k}
\end{array}\right)=\left(\begin{array}{cc}
2 Q O_{3} & -\frac{1}{2} Q O_{2} \\
2 Q O_{2} & -\frac{1}{2} Q O_{1}
\end{array}\right),
\end{aligned}
$$$$
M^{3} \cdot Q_{O}=\left(\begin{array}{cc}
\frac{1}{2}+\frac{5}{16} \vec{i}+\frac{3}{16} \vec{j}+\frac{7}{64} \vec{k} & -\frac{3}{16}-\frac{1}{8} \vec{i}-\frac{5}{64} \vec{j}-\frac{3}{64} \vec{k} \\
\frac{3}{4}+\frac{1}{2} \vec{i}+\frac{5}{16} \vec{j}+\frac{3}{16} \vec{k} & -\frac{1}{4}-\frac{3}{16} \vec{i}-\frac{1}{8} \vec{j}-\frac{5}{64} \vec{k}
\end{array}\right)=\left(\begin{array}{cc}
2 Q O_{4} & -\frac{1}{2} Q O_{3} \\
2 Q O_{3} & -\frac{1}{2} Q O_{2}
\end{array}\right),
$$

$M^{4} \cdot Q_{O}=\left(\begin{array}{cc}\frac{5}{16}+\frac{3}{16} \vec{i}+\frac{7}{64} \vec{j}+\frac{1}{16} \vec{k} & -\frac{1}{8}-\frac{5}{64} \vec{i}-\frac{3}{64} \vec{j}-\frac{7}{256} \vec{k} \\ \frac{1}{2}+\frac{5}{16} \vec{i}+\frac{3}{16} \vec{j}+\frac{7}{64} \vec{k} & -\frac{3}{16}-\frac{1}{8} \vec{i}-\frac{5}{64} \vec{j}-\frac{3}{64} \vec{k}\end{array}\right)=\left(\begin{array}{cc}2 Q O_{5} & -\frac{1}{2} Q O_{4} \\ 2 Q O_{4} & -\frac{1}{2} Q O_{3}\end{array}\right) . \mathrm{Em}$

seguida, vejamos que $M^{-1} Q_{M}=\left(\begin{array}{cc}0+\vec{i}+\vec{j}+\frac{3}{4} \vec{k} & 1+0 \vec{i}-\frac{1}{4} \vec{j}-\frac{1}{4} \vec{k} \\ -4+0 \vec{i}+\vec{j}+\vec{k} & 4+\vec{i}+0 \vec{j}-\frac{1}{4} \vec{k}\end{array}\right)=\left(\begin{array}{cc}2 Q O_{0} & -\frac{1}{2} Q O_{-1} \\ 2 Q O_{-1} & -\frac{1}{2} Q O_{-2}\end{array}\right)$,

$M^{-2} \cdot Q_{O}=\left(\begin{array}{cc}-4+0 \vec{i}+\vec{j}+\vec{k} & 4+1 \vec{i}+0 \vec{j}-\frac{1}{4} \vec{k} \\ -16-4 \vec{i}+0 \vec{j}+\vec{k} & 12+4 \vec{i}+\vec{j}+0 \vec{k}\end{array}\right)=\left(\begin{array}{cc}2 Q O_{-1} & -\frac{1}{2} Q O_{-2} \\ 2 Q O_{-2} & -\frac{1}{2} Q O_{-3}\end{array}\right)$,

$M^{-3} \cdot Q_{O}=\left(\begin{array}{cc}-16-4 i+0 j+k & 12+4 i+j+0 k \\ -48-16 i-4 j+0 k & 32+12 i+4 j+k\end{array}\right)=\left(\begin{array}{cc}2 Q O_{-2} & -\frac{1}{2} Q O_{-3} \\ 2 Q O_{-3} & -\frac{1}{2} Q O_{-4}\end{array}\right)$, etc.

Com origem no comportamento dos produtos indicados das matrizes por $\left(M^{n} \cdot Q_{O}\right)$ e também por $\left(M^{-n} \cdot Q_{O}\right)$ enunciaremos o teorema seguinte.

Teorema 8: Temos as seguintes propriedades para as matrizes: $Q M^{n}=\left(\begin{array}{cc}2 Q O_{n+1} & -\frac{1}{2} Q O_{n} \\ 2 Q O_{n} & -\frac{1}{2} Q O_{n-1}\end{array}\right)=M(n) \cdot Q_{O}=\left(\begin{array}{cc}1 & -\frac{1}{4} \\ 1 & 0\end{array}\right)^{n}\left(\begin{array}{cc}2 Q_{1} & -\frac{1}{2} Q_{0} \\ 2 Q_{0} & -\frac{1}{2} Q_{-1}\end{array}\right)$ 
$Q M^{-n}=Q \cdot M(-n)=\left(\begin{array}{cc}2 Q O_{-n+1} & -\frac{1}{2} Q O_{-n} \\ 2 Q O_{-n} & -\frac{1}{2} Q O_{-n-1}\end{array}\right)=\left(\begin{array}{cc}1 & -\frac{1}{4} \\ 1 & 0\end{array}\right)^{-n} \cdot Q_{O}$

(iii) $Q O_{n}^{2}-Q O_{n+1} \cdot Q O_{n-1}=-\frac{1}{\left(-2^{2 n}\right)}\left(\frac{43}{64}+\frac{15}{16} \vec{i}+\frac{3}{4} \vec{j}+0 \vec{k}\right)$;

(iv) $Q M_{Q, k}^{n}=\left(\begin{array}{cc}2 Q O_{k, n+1} & -\frac{1}{2} Q O_{k, n} \\ 2 Q O_{k, n} & -\frac{1}{2} Q O_{k, n-1}\end{array}\right)=\left(\begin{array}{cc}1 & -\frac{1}{4} \\ 1 & 0\end{array}\right)^{n} \cdot\left(\begin{array}{cc}2 Q_{k, 1} & -\frac{1}{2} Q_{k, 0} \\ 2 Q_{k .0} & -\frac{1}{2} Q_{k,-1}\end{array}\right)$;

(v) $Q O_{k, n}{ }^{2}-Q O_{k, n+1} \cdot Q O_{k, n-1}=-\frac{1}{\left(-2^{2 n}\right)}\left(-\frac{k^{6}-k^{4}-4 k^{2}+3}{k^{4}}+\frac{k^{4}-1}{k^{4}} \vec{i}+\frac{3 k^{2}-1}{k^{2}} \vec{j}+\frac{3 k^{2}-4}{k^{2}} \vec{k}\right)$,

Para todo inteiro $k \geq 3$.

Prova. De imediato, vamos considerar a seguinte matriz indicada pelo produto de matrizes

$Q M^{n}=\left(\begin{array}{cc}2 Q O_{n+1} & -\frac{1}{2} Q O_{n} \\ 2 Q O_{n} & -\frac{1}{2} Q O_{n-1}\end{array}\right)=\left(\begin{array}{cc}2\left(O_{n+1}+O_{n+2} \vec{i}+O_{n+3} \vec{j}+O_{n+4} \vec{k}\right) & -\frac{1}{2}\left(O_{n}+O_{n+1} \vec{i}+O_{n+2} \vec{j}+O_{n+3} \vec{k}\right) \\ 2\left(O_{n}+O_{n+1} \vec{i}+O_{n+2} \vec{j}+O_{n+3} \vec{k}\right) & -\frac{1}{2}\left(O_{n-1}+O_{n} \vec{i}+O_{n+1} \vec{j}+O_{n+2} \vec{k}\right)\end{array}\right)$

$=\left(\begin{array}{cc}2 O_{n+1} & -\frac{1}{2} O_{n} \\ 2 O_{n} & -\frac{1}{2} O_{n-1}\end{array}\right)+\left(\begin{array}{cc}2 O_{n+2} & -\frac{1}{2} O_{n+1} \\ 2 O_{n+1} & -\frac{1}{2} O_{n}\end{array}\right) \cdot \vec{i}+\left(\begin{array}{cc}2 O_{n+3} & -\frac{1}{2} O_{n+2} \\ 2 O_{n+2} & -\frac{1}{2} O_{n+1}\end{array}\right) \cdot \vec{j}+\left(\begin{array}{cc}2 O_{n+4} & -\frac{1}{2} O_{n+3} \\ 2 O_{n+3} & -\frac{1}{2} O_{n+2}\end{array}\right) \cdot \vec{k}=$

$=M^{n}+M^{n+1} \vec{i}+M^{n+2} \vec{j}+M^{n+3} \vec{k}=M^{n}+M^{n} M \vec{i}+M^{n} M^{2} \vec{j}+M^{n} M^{3} \vec{k}=M^{n}\left(I+M \vec{i}+M^{2} \vec{j}+M^{3} \vec{k}\right)=$

$=\left(\begin{array}{cc}1 & -\frac{1}{4} \\ 1 & 0\end{array}\right)^{n}=\left[\left(\begin{array}{cc}1 & 0 \\ 0 & 1\end{array}\right)+\left(\begin{array}{cc}1 & -\frac{1}{4} \\ 1 & 0\end{array}\right) \vec{i}+\left(\begin{array}{cc}1 & -\frac{1}{4} \\ 1 & 0\end{array}\right)^{2} \vec{j}+\left(\begin{array}{cc}1 & -\frac{1}{4} \\ 1 & 0\end{array}\right)^{3} \vec{k}\right]=\left(\begin{array}{cc}1 & -\frac{1}{4} \\ 1 & 0\end{array}\right)^{n}\left(\begin{array}{cc}2 O_{1} & -\frac{1}{2} O_{0} \\ 2 O_{0} & -\frac{1}{2} O_{-1}\end{array}\right)=$

$=\left(\begin{array}{cc}1 & -\frac{1}{4} \\ 1 & 0\end{array}\right)^{n}\left[\left(\begin{array}{ll}1 & 0 \\ 0 & 1\end{array}\right)+\left(\begin{array}{cc}2 O_{2} & -\frac{1}{2} O_{1} \\ 2 O_{1} & -\frac{1}{2} O_{0}\end{array}\right) \vec{i}+\left(\begin{array}{cc}2 O_{3} & -\frac{1}{2} O_{2} \\ 2 O_{2} & -\frac{1}{2} O_{1}\end{array}\right) \vec{j}+\left(\begin{array}{cc}2 O_{4} & -\frac{1}{2} O_{3} \\ 2 O_{3} & -\frac{1}{2} O_{2}\end{array}\right) \vec{k}\right]=M(n) \cdot Q_{O}$, visto

que $\left(\begin{array}{cc}2 \cdot O_{1} & -\frac{1}{2} O_{0} \\ 2 \cdot O_{0} & -\frac{1}{2} O_{-1}\end{array}\right)+\left(\begin{array}{cc}2 O_{2} & -\frac{1}{2} O_{1} \\ 2 O_{1} & -\frac{1}{2} O_{0}\end{array}\right) \vec{i}+\left(\begin{array}{cc}2 O_{3} & -\frac{1}{2} O_{2} \\ 2 O_{2} & -\frac{1}{2} O_{1}\end{array}\right) \vec{j}+\left(\begin{array}{cc}2 O_{4} & -\frac{1}{2} O_{3} \\ 2 O_{3} & -\frac{1}{2} O_{2}\end{array}\right) \vec{k}=\left(\begin{array}{cc}2 Q_{1} & -\frac{1}{2} Q_{0} \\ 2 Q_{0} & -\frac{1}{2} Q_{-1}\end{array}\right)$.

Finalmente, desde que encontramos o comportamento para o determinante da expressão $Q O_{n}{ }^{2}-Q O_{n+1} \cdot Q O_{n-1}=\operatorname{det}\left(\begin{array}{cc}1 & -\frac{1}{4} \\ 1 & 0\end{array}\right)^{n} \cdot \operatorname{det}\left(\begin{array}{cc}2 Q_{1} & -\frac{1}{2} Q_{0} \\ 2 Q_{0} & -\frac{1}{2} Q_{-1}\end{array}\right)=-\frac{1}{\left(-2^{2 n}\right)}\left(\frac{125}{64}+\frac{15}{16} \vec{i}+\frac{3}{4} \vec{j}+0 \vec{k}\right)$. 
De forma semelhante, para o último item, vamos determinar o comportamento do determinante $\operatorname{det}\left(\begin{array}{cc}2 Q O_{k, n+1} & -\frac{1}{2} Q O_{k, n} \\ 2 Q O_{k, n} & -\frac{1}{2} Q O_{k, n-1}\end{array}\right)=\operatorname{det}\left(\begin{array}{cc}1 & -\frac{1}{4} \\ 1 & 0\end{array}\right)^{n} \cdot \operatorname{det}\left(\begin{array}{cc}2 Q_{k, 1} & -\frac{1}{2} Q_{k, 0} \\ 2 Q_{k, 0} & -\frac{1}{2} Q_{k,-1}\end{array}\right)$

Verificamos, também, as propriedades das seguintes somatórias finitas.

Teorema 9: Valem as seguintes relações para as somatórias dos números de Oresme e os kNúmeros de Oresme: (i) $\frac{n}{4} \cdot \sum_{i=0}^{n-1} Q O_{i}=\frac{1}{2}-Q O_{n+1}$; (ii) $\frac{n}{k^{2}} \cdot \sum_{i=0}^{n-1} Q O_{k, i}=\frac{1}{2}-Q O_{k, n+1}$.

Prova. De imediato, a partir do teorema, vamos tomar as seguintes igualdades $Q O_{2}=Q O_{1}-\frac{1}{4} Q O_{0}$ $Q O_{3}=Q O_{2}-\frac{1}{4} Q O_{1}$ $\left\{\mathrm{OO}_{4}=Q_{3}-\frac{1}{4} Q \mathrm{O}_{2}\right.$. De imediato, efetuando o cancelamento de termos correspondentes, $Q O_{n+1}=Q O_{n}-\frac{1}{4} Q O_{n-1}$

determinamos que $Q O_{n+1}-Q O_{1}=-\frac{n}{4} \cdot \sum_{i=0}^{n-1} Q O_{i}$. De forma semelhante, se considerarmos os kQuaternions de Oresme, devemos verificar que $Q O_{k, n+1}-Q O_{k, 1}=-\frac{n}{k^{2}} \cdot \sum_{i=0}^{n-1} Q O_{k, i}$.

Teorema 10: A função geradora correspondente os Quaternions de Oresme e aos k-Quaternions de Oresme são, respectivamente, dadas pelas seguintes expressões formais correspondentes $g_{Q}(x)=\frac{Q O_{0}+\left(Q O_{1}-Q O_{0}\right) x}{1-x+\frac{1}{4} x^{2}}$ e $g_{Q, k}(x)=\frac{Q O_{k, 0}+\left(Q O_{k, 1}-Q O_{k, 0}\right) x}{1-x+\frac{1}{k^{2}} x^{2}}$.

Prova. Recordando a seguinte recorrência fundamental $Q O_{k, n+2}-Q O_{k, n+1}+\frac{1}{4} Q O_{k, n}=0, n \geq 0$. Em seguida, vamos tomar as seguintes somas infinitas formais descritas por $g_{Q}(x)=\sum_{i=0}^{\infty} Q O_{i} \cdot x^{i} \mathrm{e}$ $g_{Q, k}(x)=\sum_{i=0}^{\infty} Q O_{k, i} \cdot x^{i}$. Vemos que: 


$$
\left\{\begin{array}{l}
g_{Q}(x)=Q O_{0}+Q O_{1} x+Q O_{2} x^{2}+Q O_{3} x^{3}+Q O_{4} x^{4}+Q O_{5} x^{5} \cdots+Q O_{n} x^{n}+ \\
-x \cdot g_{Q}(x)=-Q O_{0} x-Q O_{1} x^{2}-Q O_{2} x^{3}-\cdots-Q O_{n-1} x^{n}-Q O_{n} x^{n+1}-Q O_{n+1} x^{n+2}-\cdots \\
\frac{1}{4} x^{2} \cdot g_{Q}(x)=\frac{1}{4} Q O_{0} x^{2}+\frac{1}{4} Q O_{1} x^{3}+\frac{1}{4} Q O_{2} x^{4}+\cdots+\frac{1}{4} Q O_{n-2} x^{n}+\frac{1}{4} Q O_{n-1} x^{n+1}+\cdots
\end{array}\right.
$$

No passo seguinte, vamos tomar que $\left(g(x)-x \cdot g(x)+\frac{1}{4} x^{2} \cdot g(x)\right)=Q O_{0}+\left(Q O_{1}-Q O_{0}\right) x+$ $+\left(Q O_{2}-Q O_{1}+\frac{1}{4} Q O_{0}\right) x^{2}+\cdots+\left(Q O_{n+2}-Q O_{n+1}+\frac{1}{4} Q O_{n}\right) x^{2}+\cdots=Q O_{0}+\left(Q O_{1}-Q O_{0}\right) x$. Por conseguinte, determinamos que $g_{Q}(x)-x \cdot g_{Q}(x)+\frac{1}{4} x^{2} \cdot g_{Q}(x)=Q O_{0}+\left(Q O_{1}-Q O_{0}\right) x$.

\section{Os Octônions de Oresme}

A partir da literatura especializada, constatamos o interesse de representações por intermédio dos Octônions para algumas sequências recorrentes, todavia, não registramos resultados correspondentes aos números de Oresme. Assim, com origem em determinados argumentos indicados nas seções predecessoras, vamos introduzir a seguinte definição que corresponde a uma nova noção introduzida no presente trabalho.

Definição 6: Chamamos de Octonion de Oresme de ordem ' $\mathrm{n}$ ' ao elemento descrito por $\Theta_{n}=O_{n} i_{0}+O_{n+1} i_{1}+O_{n+2} i_{2}+O_{n+3} i_{3}+O_{n+4} i_{4}+O_{n+5} i_{5}+O_{n+6} i_{6}+O_{n+7} i_{7}=\sum_{s=0}^{7} O_{n+s} i_{s}$, em que os coeficientes são os números de Oresme e as unidades imaginárias são descritas pelas operações formais $i_{0}{ }^{2}=i_{1}^{2}=i_{2}^{2}=i_{3}^{2}=i_{4}^{2}=i_{5}^{2}=i_{6}^{2}=i_{7}^{2}=-1$.

Podemos verificar que ocorrem: $\Theta_{-1}=-2 i_{0}+0 i_{1}+\frac{1}{2} i_{2}+\frac{2}{4} i_{3}+\frac{3}{8} i_{4}+\frac{4}{16} i_{5}+\frac{5}{32} i_{6}+\frac{6}{64} i_{7}$, $\Theta_{0}=0 i_{0}+\frac{1}{2} i_{1}+\frac{2}{4} i_{2}+\frac{3}{8} i_{3}+\frac{4}{16} i_{4}+\frac{5}{32} i_{5}+\frac{6}{64} i_{6}+\frac{7}{128} i_{7}, \Theta_{1}=\frac{1}{2} i_{0}+\frac{2}{4} i_{1}+\frac{3}{8} i_{2}+\frac{4}{16} i_{3}+\frac{5}{32} i_{4}$ $+\frac{6}{64} i_{5}+\frac{7}{128} i_{6}+\frac{8}{256} i_{7}$

Definição 7: Chamamos de Octonion de k-Oresme de ordem 'n' ao elemento descrito por $\Theta_{k, n}=O_{k, n}+O_{k, n+1} i_{0}+O_{k, n+2} i_{1}+O_{k, n+3} i_{2}+O_{k, n+4} i_{3}+O_{k, n+5} i_{4}+O_{k, n+6} i_{5}+O_{k, n+7} i_{6}+O_{k, n+8} i_{7}$, em que os coeficientes são os números de Oresme e as unidades imaginárias são descritas pelas operações formais com as unidades indicadas $i_{0}{ }^{2}=i_{1}{ }^{2}=i_{2}{ }^{2}=i_{3}{ }^{2}=i_{4}{ }^{2}=i_{5}{ }^{2}=i_{6}{ }^{2}=i_{7}{ }^{2}=-1$.

Decorrem, de forma imediata, as seguintes relações para os Octônions de Oresme.

Teorema 11: (i) $\Theta_{n+2}=\Theta_{n+1}-\frac{1}{4} \Theta_{n}$ e $\Theta_{k, n+2}=\Theta_{k, n+1}-\frac{1}{4} \Theta_{k, n}$;

(ii) $\Theta_{n}=\frac{1}{2^{n}}\left(n e_{0}+\frac{n+1}{2} i_{1}+\frac{n+2}{4} i_{2}+\frac{n+3}{8} i_{3}+\frac{n+4}{16} i_{4}+\frac{n+5}{32} i_{5}+\frac{n+6}{64} i_{6}+\frac{n+7}{128} i_{7}\right)$; 
(iii) $\Theta_{-n}=-2^{n}\left(n e_{0}+\frac{n-1}{2} i_{1}+\frac{n-2}{4} i_{2}+\frac{n-3}{8} i_{3}+\frac{n-4}{16} i_{4}+\frac{n-5}{32} i_{5}+\frac{n-6}{64} i_{6}+\frac{n-7}{128} i_{7}\right)$.

Prova. Basta ver que $\Theta_{n+1}-\frac{1}{4} \Theta_{n}=\sum_{s=0}^{8} O_{n+1+s} i_{s}-\frac{1}{4} \sum_{s=0}^{8} O_{n+s} i_{s}=\sum_{s=0}^{8}\left(O_{n+1+s}-\frac{1}{4} O_{n+s}\right) \cdot i_{s}=\Theta_{n+2}$.

Para os demais itens, repetimos os argumentos do teorema 7.

Vamos, agora, definir as seguintes matrizes determinadas pelos Octônions a seguir $\Theta M(n)=\left(\begin{array}{cc}2 \Theta O_{n+1} & -\frac{1}{2} \Theta O_{n} \\ 2 \Theta O_{n} & -\frac{1}{2} \Theta O_{n-1}\end{array}\right)$ e $\Theta M_{k}(n)=\left(\begin{array}{cc}2 \Theta O_{k, n+1} & -\frac{1}{2} \Theta O_{k, n} \\ 2 \Theta O_{k, n} & -\frac{1}{2} \Theta O_{k, n-1}\end{array}\right)$. E, em seguida, po-

demos examinar as seguintes decomposições que verificamos apenas substituindo os termos

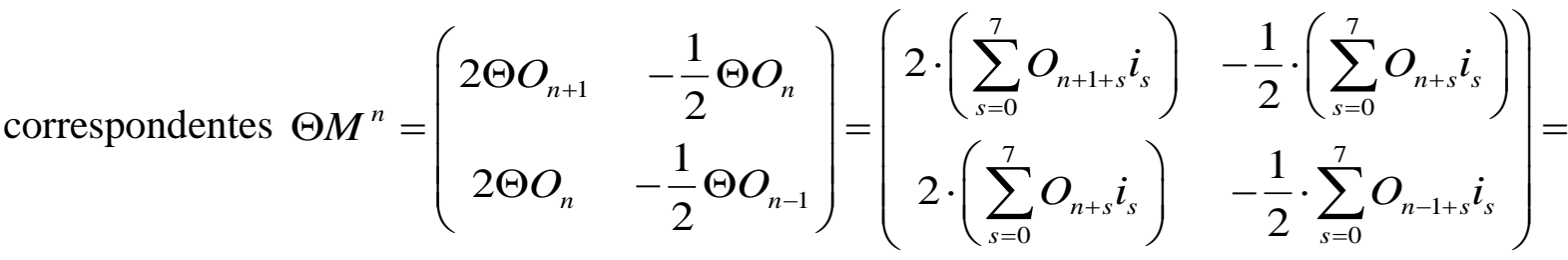
$=Q M^{n} i_{0}+Q M^{n+1} i_{1}+Q M^{n+2} i_{2}+Q M^{n+3} i_{3}+Q M^{n+4} i_{4}+Q M^{n+5} i_{5}+Q M^{n+6} i_{6}+Q M^{n+7} i_{7}=$ $=\left(\begin{array}{cc}1 & -\frac{1}{4} \\ 1 & 0\end{array}\right)^{n}\left(\begin{array}{cc}2 O_{1} & -\frac{1}{2} O_{0} \\ 2 O_{0} & -\frac{1}{2} O_{-1}\end{array}\right) i_{0}+\left(\begin{array}{cc}1 & -\frac{1}{4} \\ 1 & 0\end{array}\right)^{n+1}\left(\begin{array}{cc}2 O_{1} & -\frac{1}{2} O_{0} \\ 2 O_{0} & -\frac{1}{2} O_{-1}\end{array}\right) i_{1}+\left(\begin{array}{cc}1 & -\frac{1}{4} \\ 1 & 0\end{array}\right)^{n+2}\left(\begin{array}{cc}2 O_{1} & -\frac{1}{2} O_{0} \\ 2 O_{0} & -\frac{1}{2} O_{-1}\end{array}\right) i_{2}+$ $\left(\begin{array}{cc}1 & -\frac{1}{4} \\ 1 & 0\end{array}\right)^{n+3}\left(\begin{array}{cc}2 O_{1} & -\frac{1}{2} O_{0} \\ 2 O_{0} & -\frac{1}{2} O_{-1}\end{array}\right) i_{3}+\left(\begin{array}{cc}1 & -\frac{1}{4} \\ 1 & 0\end{array}\right)^{n+4}\left(\begin{array}{cc}2 O_{1} & -\frac{1}{2} O_{0} \\ 2 O_{0} & -\frac{1}{2} O_{-1}\end{array}\right) i_{4}+\left(\begin{array}{cc}1 & -\frac{1}{4} \\ 1 & 0\end{array}\right)^{n+5}\left(\begin{array}{cc}2 O_{1} & -\frac{1}{2} O_{0} \\ 2 O_{0} & -\frac{1}{2} O_{-1}\end{array}\right) i_{5}+$ $+\left(\begin{array}{cc}1 & -\frac{1}{4} \\ 1 & 0\end{array}\right)^{n+6}\left(\begin{array}{cc}2 O_{1} & -\frac{1}{2} O_{0} \\ 2 O_{0} & -\frac{1}{2} O_{-1}\end{array}\right) i_{6}+\left(\begin{array}{cc}1 & -\frac{1}{4} \\ 1 & 0\end{array}\right)^{n+7}\left(\begin{array}{cc}2 O_{1} & -\frac{1}{2} O_{0} \\ 2 O_{0} & -\frac{1}{2} O_{-1}\end{array}\right) i_{7}=\left(\begin{array}{cc}1 & -\frac{1}{4} \\ 1 & 0\end{array}\right)^{n}\left(\begin{array}{cc}2 \Theta_{1} & -\frac{1}{2} \Theta_{0} \\ 2 \Theta_{0} & -\frac{1}{2} \Theta_{-1}\end{array}\right)$.

Novamente, encontramos uma decomposição para a matriz determinada pelos octônions de Oresme a partir do produto de matriz elementares do tipo $\left(\begin{array}{cc}1 & -\frac{1}{4} \\ 1 & 0\end{array}\right)^{n} \cdot\left(\begin{array}{cc}2 \Theta_{1} & -\frac{1}{2} \Theta_{0} \\ 2 \Theta_{0} & -\frac{1}{2} \Theta_{-1}\end{array}\right)$. Enunciaremos, pois, o seguinte teorema, recordando que temos a matriz $\left(\begin{array}{cc}2 \Theta_{1} & -\frac{1}{2} \Theta_{0} \\ 2 \Theta_{0} & -\frac{1}{2} \Theta_{-1}\end{array}\right)=$

$$
\left(\begin{array}{ccc}
2\left(\frac{1}{2} i_{0}+\frac{2}{4} i_{1}+\frac{3}{8} i_{2}+\frac{4}{16} i_{3}+\frac{5}{32} i_{4}+\frac{6}{64} i_{5}+\frac{7}{128} i_{6}+\frac{8}{256} i_{7}\right) & -\frac{1}{2}\left(0 i_{0}+\frac{1}{2} i_{1}+\frac{2}{4} i_{2}+\frac{3}{8} i_{3}+\frac{4}{16} i_{4}+\frac{5}{32} i_{5}+\frac{6}{64} i_{6}+\frac{7}{128} i_{7}\right) \\
2\left(0 i_{0}+\frac{1}{2} i_{1}+\frac{2}{4} i_{2}+\frac{3}{8} i_{3}+\frac{4}{16} i_{4}+\frac{5}{32} i_{5}+\frac{6}{64} i_{6}+\frac{7}{128} i_{7}\right) & -\frac{1}{2}\left(-2 i_{0}+0 i_{1}+\frac{1}{2} i_{2}+\frac{2}{4} i_{3}+\frac{3}{8} i_{4}+\frac{4}{16} i_{5}+\frac{5}{32} i_{6}+\frac{6}{64} i_{7}\right)
\end{array}\right)
$$

ALVES, F. R. V. Sequência de Oresme e algumas propriedades (matriciais) generalizadas. C.Q.D.- Revista Eletrônica Paulista de Matemática, Bauru, v. 16, p. 28-52, dez. 2019 
Teorema 12: Valem as seguintes relações matriciais.

(i) $\Theta M^{n}=\left(\begin{array}{cc}2 \Theta O_{n+1} & -\frac{1}{2} \Theta O_{n} \\ 2 \Theta O_{n} & -\frac{1}{2} \Theta O_{n-1}\end{array}\right)=\left(\begin{array}{cc}1 & -\frac{1}{4} \\ 1 & 0\end{array}\right)^{n} \cdot\left(\begin{array}{cc}2 \Theta_{1} & -\frac{1}{2} \Theta_{0} \\ 2 \Theta_{0} & -\frac{1}{2} \Theta_{-1}\end{array}\right)$;

(ii) $\Theta M^{-n}=\left(\begin{array}{cc}2 \Theta O_{n+1} & -\frac{1}{2} \Theta O_{n} \\ 2 \Theta O_{n} & -\frac{1}{2} \Theta O_{n-1}\end{array}\right)=\left(\begin{array}{cc}1 & -\frac{1}{4} \\ 1 & 0\end{array}\right)^{-n}\left(\begin{array}{cc}2 \Theta_{1} & -\frac{1}{2} \Theta_{0} \\ 2 \Theta_{0} & -\frac{1}{2} \Theta_{-1}\end{array}\right)$; (iii) $\operatorname{det}\left(\begin{array}{cc}2 \Theta O_{n+1} & -\frac{1}{2} \Theta O_{n} \\ 2 \Theta O_{n} & -\frac{1}{2} \Theta O_{n-1}\end{array}\right)=$

$=\frac{1}{\left(-2^{2 n}\right)}\left(\frac{1366191}{16384} i_{0}+\frac{225}{16} i_{1}-\frac{709}{8} i_{2}-\frac{637}{16} i_{3}+\frac{1995}{8} i_{4}+\frac{3081}{16} i_{5}+269 i_{6}+\frac{7517}{16} i_{7}\right)$.

Prova. Reparemos que podemos verificar o resultado por intermédio de indução. De fato, assumindo que temos a igualdade $\left(\begin{array}{cc}2 \Theta O_{n+1} & -\frac{1}{2} \Theta O_{n} \\ 2 \Theta O_{n} & -\frac{1}{2} \Theta O_{n-1}\end{array}\right)=\left(\begin{array}{cc}1 & -\frac{1}{4} \\ 1 & 0\end{array}\right)^{n}\left(\begin{array}{cc}2 \Theta_{1} & -\frac{1}{2} \Theta_{0} \\ 2 \Theta_{0} & -\frac{1}{2} \Theta_{-1}\end{array}\right)$. Verificare$\operatorname{mos}$ que $\left(\begin{array}{cc}1 & -\frac{1}{4} \\ 1 & 0\end{array}\right)^{n+1}\left(\begin{array}{cc}2 \Theta_{1} & -\frac{1}{2} \Theta_{0} \\ 2 \Theta_{0} & -\frac{1}{2} \Theta_{-1}\end{array}\right)=\left(\begin{array}{cc}1 & -\frac{1}{4} \\ 1 & 0\end{array}\right)\left(\begin{array}{cc}1 & -\frac{1}{4} \\ 1 & 0\end{array}\right)^{n}\left(\begin{array}{cc}2 \Theta_{1} & -\frac{1}{2} \Theta_{0} \\ 2 \Theta_{0} & -\frac{1}{2} \Theta_{-1}\end{array}\right)=\left(\begin{array}{cc}1 & -\frac{1}{4} \\ 1 & 0\end{array}\right)\left(\begin{array}{cc}2 \Theta O_{n+1} & -\frac{1}{2} \Theta O_{n} \\ 2 \Theta O_{n} & -\frac{1}{2} \Theta O_{n-1}\end{array}\right)$ $=\left(\begin{array}{cc}2 \Theta O_{n+2} & -\frac{1}{2} \Theta O_{n+1} \\ 2 \Theta O_{n+1} & -\frac{1}{2} \Theta O_{n}\end{array}\right)=\Theta M^{n+1}$. Para o último item, basta determinar os respectivos determinantes .

Teorema 13: Valem as seguintes relações para os Octônions de Oresme: $\frac{n}{4} \sum_{i=0}^{n-1} \Theta_{i}=\Theta_{1}-\Theta_{n+1} ;$ (ii) $\frac{n}{k^{2}} \sum_{i=0}^{n-1} \Theta_{k, i}=\Theta_{k, 1}-\Theta_{k, n+1}$; (iii) 
Vamos considerar a seguintes expressões $\left\{\begin{array}{l}\Theta_{2}=\Theta_{1}-\frac{1}{4} \Theta_{0} \\ \Theta_{3}=\Theta_{2}-\frac{1}{4} \Theta_{1} \\ \Theta_{4}=\Theta_{3}-\frac{1}{4} \Theta_{2} \quad . \text { Efetuando o cancelamento dos } \\ \vdots \\ \Theta_{n+1}=\Theta_{n}-\frac{1}{4} \Theta_{n-1}\end{array}\right.$ termos correspondentes, encontraremos que $\Theta_{n+1}-\Theta_{1}=-\frac{n}{4} \sum_{i=0}^{n-1} \Theta_{i}$.

Teorema 14: A função geradora correspondente os Octônions de Oresme e aos k-Octônions de Oresme são, respectivamente, dadas pelas seguintes expressões racionais indicadas $g_{\Theta}(x)=\frac{\Theta O_{0}+\left(\Theta O_{1}-\Theta O_{0}\right) x}{1-x+\frac{1}{4} x^{2}}$ e $g_{\Theta, k}(x)=\frac{\Theta O_{k, 0}+\left(\Theta O_{k, 1}-\Theta O_{k, 0}\right) x}{1-x+\frac{1}{k^{2}} x^{2}}$.

Prova. Recordando a seguinte recorrência fundamental entre os octônions $\Theta O_{k, n+2}-\Theta O_{k, n+1}+\frac{1}{4} \Theta O_{k, n}=0, n \geq 0$. Em seguida, vamos tomar as seguintes somas infinitas formais $g_{\Theta}(x)=\sum_{i=0}^{\infty} \Theta O_{i} \cdot x^{i}$ e $g_{\Theta, k}(x)=\sum_{i=0}^{\infty} \Theta O_{k, i} \cdot x^{i}$.

\section{Os Sedenions de Oresme}

Bilgici, Tokeser e Unal (2017) explicam que uma Álgebra de Sedenions, denotata por $S$ é uma Álgebra de Cauley-Dicson de dimensão 16. De modo simplificado, podemos considerar a seguinte bases para os seus elementos que os autores indicam por $\left\{e_{0}, e_{1}, e_{2}, \ldots, e_{15}\right\}$ e que um Sedênion pode ser descrito da forma $s=\sum_{i=0}^{15} a_{i} e_{i}$ onde $a_{0}, a_{1}, a_{2}, \ldots, a_{15} \in I R$. Para exemplificar, trazemos abaixo uma tabela que descreve as operações formais envolvendo os elementos da base indicada por $\left\{e_{0}, e_{1}, e_{2}, e_{3}, e_{4}, e_{5} \ldots, e_{15}\right\}$. 


\begin{tabular}{r|rrrrrrrrrrrrrrrr}
\hline & 0 & 1 & 2 & 3 & 4 & 5 & 6 & 7 & 8 & 9 & 10 & 11 & 12 & 13 & 14 & 15 \\
\hline 0 & 0 & 1 & 2 & 3 & 4 & 5 & 6 & 7 & 8 & 9 & 10 & 11 & 12 & 13 & 14 & 15 \\
1 & 1 & -0 & 3 & -2 & 5 & -4 & -7 & 6 & 9 & -8 & -11 & 10 & -13 & 12 & 15 & -14 \\
2 & 2 & -3 & -0 & 1 & 6 & 7 & -4 & -5 & 10 & 11 & -8 & -9 & -14 & -15 & 12 & 13 \\
3 & 3 & 2 & -1 & -0 & 7 & -6 & 5 & -4 & 11 & -10 & 9 & -8 & -15 & 14 & -13 & 12 \\
4 & 4 & -5 & -6 & -7 & -0 & 1 & 2 & 3 & 12 & 13 & 14 & 15 & -8 & -9 & -10 & -11 \\
5 & 5 & 4 & -7 & 6 & -1 & -0 & -3 & 2 & 13 & -12 & 15 & -14 & 9 & -8 & 11 & -10 \\
6 & 6 & 7 & 4 & -5 & -2 & 3 & -0 & -1 & 14 & -15 & -12 & 13 & 10 & -11 & -8 & 9 \\
7 & 7 & -6 & 5 & 4 & -3 & -2 & 1 & -0 & 15 & 14 & -13 & -12 & 11 & 10 & -9 & -8 \\
8 & 8 & -9 & -10 & -11 & -12 & -13 & -14 & -15 & -0 & 1 & 2 & 3 & 4 & 5 & 6 & 7 \\
9 & 9 & 8 & -11 & 10 & -13 & 12 & 15 & -14 & -1 & -0 & -3 & 2 & -5 & 4 & 7 & -6 \\
10 & 10 & 11 & 8 & -9 & -14 & -15 & 12 & 13 & -2 & 3 & -0 & -1 & -6 & -7 & 4 & 5 \\
11 & 11 & -10 & 9 & 8 & -15 & 14 & -13 & 12 & -3 & -2 & 1 & -0 & -7 & 6 & -5 & 4 \\
12 & 12 & 13 & 14 & 15 & 8 & -9 & -10 & -11 & -4 & 5 & 6 & 7 & -0 & -1 & -2 & -3 \\
13 & 13 & -12 & 15 & -14 & 9 & 8 & 11 & -10 & -5 & -4 & 7 & -6 & 1 & -0 & 3 & -2 \\
14 & 14 & -15 & -12 & 13 & 10 & -11 & 8 & 9 & -6 & -7 & -4 & 5 & 2 & -3 & -0 & 1 \\
15 & 15 & 14 & -13 & -12 & 11 & 10 & -9 & 8 & -7 & 6 & -5 & -4 & 3 & 2 & -1 & -0
\end{tabular}

Figura 2. Bilgici, Tokeser e Unal (2017) descrevem os resultados das operações entre os elementos da base dos sedenions

Definição 8: Chamamos de Sedenion de Oresme de ordem 'n' ao elemento descrito por $S O_{n}=\sum_{s=0}^{15} O_{n+s} i_{s}$ em que $\left\{e_{0}, e_{1}, e_{2}, \ldots, e_{16}\right\}$ constitui a base canônica.

Definição 9: Chamamos de k-Sedenion de Oresme de ordem 'n' ao elemento descrito por $S O_{k, n}=\sum_{s=0}^{15} O_{k, n+s} e_{s}$ em que $\left\{e_{0}, e_{1}, e_{2}, \ldots, e_{15}\right\}$ constitui a base canônica.

Vejamos, pois, algumas relações de recorrência que decorrem imediatamente dessas últimas definições.

Teorema 15: Vale a seguinte relação fundamental de recorrência para os Sedenions de Oresme: (i) $S O_{n+2}=S O_{n+1}-\frac{1}{4} S O_{n}$ e $S O_{k, n+2}=S O_{k, n+1}-\frac{1}{k^{2}} S O_{n}$; (ii) $\frac{n}{4} \sum_{i=0}^{n-1} S O_{i}=S O_{1}-S O_{n+1}$ e $\frac{n}{k^{2}} \sum_{i=0}^{n-1} S O_{k, i}=S O_{k, 1}-S O_{k, n+1}$

Prova. Vemos que $S O_{n+1}-\frac{1}{4} S O_{n}=\sum_{s=0}^{15} O_{n+1+s} i_{s}-\frac{1}{4} \sum_{s=0}^{15} O_{n+s} i_{s}=\sum_{s=0}^{15}\left(O_{n+1+s}-\frac{1}{4} O_{n+s}\right) \cdot i_{s}=\sum_{s=0}^{15} O_{n+2+s} \cdot i_{s}$ $\sum_{s=0}^{15} O_{n+2+s} \cdot i_{s}=S O_{n+2}$. De forma semelhante, podemos verificar que $S O_{k, n+2}=S O_{k, n+1}-\frac{1}{k^{2}} S O_{n}$.

Para o caso dos itens (ii) e (iii) repetiremos os procedimentos abordados no teorema 4. 
Vamos, pois, definir as seguintes matrizes indicadas por $S M(n)=\left(\begin{array}{cc}2 S O_{n+1} & -\frac{1}{2} S O_{n} \\ 2 S O_{n} & -\frac{1}{2} S O_{n-1}\end{array}\right)$ e $S M_{k}(n)=\left(\begin{array}{cc}2 S O_{k, n+1} & -\frac{1}{2} S O_{k, n} \\ 2 S O_{k, n} & -\frac{1}{2} S O_{k, n-1}\end{array}\right)$. Vejamos, em seguida, o comportamento das seguintes somas $S M(n)=\left(\begin{array}{cc}2 S O_{n+1} & -\frac{1}{2} S O_{n} \\ 2 S O_{n} & -\frac{1}{2} S O_{n-1}\end{array}\right)=\left(\begin{array}{cc}2\left(\sum_{s=0}^{15} O_{n+1+s} i_{s}\right) & -\frac{1}{2}\left(\sum_{s=0}^{15} O_{n+s} i_{s}\right) \\ 2\left(\sum_{s=0}^{15} O_{n+s} i_{s}\right) & -\frac{1}{2}\left(\sum_{s=0}^{15} O_{n-1+s} i_{s}\right)\end{array}\right)=\sum_{s=0}^{15}\left(\begin{array}{cc}2 O_{n+1+s} & -\frac{1}{2} O_{n+s} \\ 2 O_{n+s} & -\frac{1}{2} O_{n-1+s}\end{array}\right) \cdot i_{s}$ $=\sum_{s=0}^{15}\left(\begin{array}{cc}2 O_{n+1+s} & -\frac{1}{2} O_{n+s} \\ 2 O_{n+s} & -\frac{1}{2} O_{n-1+s}\end{array}\right) \cdot i_{s}$. Mas, desde que conhecemos a seguinte propriedade $M(n)=\left(\begin{array}{cc}2 O_{n+1} & -\frac{1}{2} O_{n} \\ 2 O_{n} & -\frac{1}{2} O_{n-1}\end{array}\right)=\left(\begin{array}{cc}1 & -\frac{1}{4} \\ 0 & 1\end{array}\right)^{n}$. Do teorema 1 , vamos efetuar a seguinte substituição em seguida $S M(n)=\sum_{s=0}^{15}\left(\begin{array}{cc}2 O_{n+1+s} & -\frac{1}{2} O_{n+s} \\ 2 O_{n+s} & -\frac{1}{2} O_{n-1+s}\end{array}\right) \cdot i_{s}=\sum_{s=0}^{15}\left(\begin{array}{cc}1 & -\frac{1}{4} \\ 0 & 1\end{array}\right)^{n+s} \cdot i_{s}$. Seja, dessa forma, a matriz definida a partir dos Sedênions de Oresme como segue $S M=\left(\begin{array}{cc}2 S O_{1} & -\frac{1}{2} S O_{0} \\ 2 S O_{0} & -\frac{1}{2} S O_{-1}\end{array}\right)$ e o kSedênions de Oresme, como indicamos por $S M_{k}=\left(\begin{array}{cc}2 S O_{k, 1} & -\frac{1}{2} S O_{k, 0} \\ 2 S O_{k, 0} & -\frac{1}{2} S O_{k,-1}\end{array}\right)$.

Teorema 16: Valem as seguintes relações para as matrizes $S M(n)$ e $S M(-n)$. 
(i) $\quad S M(n)=\sum_{s=0}^{15}\left(\begin{array}{cc}1 & -\frac{1}{4} \\ 0 & 1\end{array}\right)^{n+s} \cdot i_{s} ; \quad$ (ii) $\quad S M(-n)=\sum_{s=0}^{15}\left(\begin{array}{cc}1 & -\frac{1}{4} \\ 0 & 1\end{array}\right)^{-n+s} \cdot i_{s}$.

$S M(n)=\left(\begin{array}{cc}2 S O_{n+1} & -\frac{1}{2} S O_{n} \\ 2 S O_{n} & -\frac{1}{2} S O_{n-1}\end{array}\right)=\left(\begin{array}{cc}1 & -\frac{1}{4} \\ 0 & 1\end{array}\right)^{n}\left(\begin{array}{cc}2 S O_{1} & -\frac{1}{2} S O_{0} \\ 2 S O_{0} & -\frac{1}{2} S O_{-1}\end{array}\right)$

$\mathrm{e}$

$S M_{\sim k}(n)=\left(\begin{array}{cc}2 S O_{k, n+1} & -\frac{1}{2} S O_{k, n} \\ 2 S O_{k, n} & -\frac{1}{2} S O_{k, n-1}\end{array}\right)=\left(\begin{array}{cc}1 & -\frac{1}{4} \\ 0 & 1\end{array}\right)^{n}\left(\begin{array}{cc}2 S O_{k, 1} & -\frac{1}{2} S O_{k, 0} \\ 2 S O_{k, 0} & -\frac{1}{2} S O_{k,-1}\end{array}\right)$.

Prova. Por indução matemática, vejamos que temos $\left(\begin{array}{cc}1 & -\frac{1}{4} \\ 0 & 1\end{array}\right)^{n+1}\left(\begin{array}{cc}2 S O_{1} & -\frac{1}{2} S O_{0} \\ 2 S O_{0} & -\frac{1}{2} S O_{-1}\end{array}\right)=$ $=\left(\begin{array}{cc}1 & -\frac{1}{4} \\ 0 & 1\end{array}\right)\left(\begin{array}{cc}1 & -\frac{1}{4} \\ 0 & 1\end{array}\right)^{n}\left(\begin{array}{cc}2 S O_{1} & -\frac{1}{2} S O_{0} \\ 2 S O_{0} & -\frac{1}{2} S O_{-1}\end{array}\right)=\left(\begin{array}{cc}1 & -\frac{1}{4} \\ 0 & 1\end{array}\right)\left(\begin{array}{cc}2 S O_{n+1} & -\frac{1}{2} S O_{n} \\ 2 S O_{n} & -\frac{1}{2} S O_{n-1}\end{array}\right)=\left(\begin{array}{cc}2 S O_{n+2} & -\frac{1}{2} S O_{n+1} \\ 2 S O_{n+1} & -\frac{1}{2} S O_{n}\end{array}\right)$

Teorema 17: A função geradora correspondente os Sedenions de Oresme e aos k-Sedenions de Oresme são, respectivamente, dadas pelas seguintes expressões algébricas racionais $g_{S}(x)=\frac{S O_{0}+\left(S O_{1}-S O_{0}\right) x}{1-x+\frac{1}{4} x^{2}}$ e $g_{S, k}(x)=\frac{S O_{k, 0}+\left(S O_{k, 1}-S O_{k, 0}\right) x}{1-x+\frac{1}{k^{2}} x^{2}}$.

Prova. Recordando a seguinte recorrência fundamental $S O_{k, n+2}-S O_{k, n+1}+\frac{1}{4} S O_{k, n}=0, n \geq 0$ . Em seguida, vamos tomar as seguintes somas infinitas formais $g_{S}(x)=\sum_{i=0}^{\infty} S O_{i} \cdot x^{i} \mathrm{e}$ $g_{S, k}(x)=\sum_{i=0}^{\infty} S O_{k, i} \cdot x^{i}$. Repetiremos os mesmos procedimentos apresentados nos teoremas.

Outras propriedades recentes concernentes aos Sedênions podem ser consultadas em Catarino (2018) ou Soykan (2019), todavia, relacionadas aos Sedênions de Fibonacci, Lucas, k-Pell e kPell-Lucas podem ser consultadas.

\section{Conclusão}

Nas seções passadas apresentamos novas relações, teoremas e propriedades derivadas de um processo de generalização dos números de Oresme. Visto que em recentes trabalhos introduzidos na literatura sobre os k-números de Oresme e os polinômios de Oresme, apresentamos propriedades e exibimos representações matriciais até o momento não discutidas na literatura especializada concernente aos Quaternions, Octônions e Sedenions de Oresme. Idem para os kQuaternions, k-Octônions e, inclusive, os k-Sedenions de Oresme. 


\section{Referências}

BILGICI, Goksal; TOKESER, Umit; UNAL, Zafer. Fibonacci and Lucas sedenions. Journal of Integer Sequences. v. 20, p. 1-12, 2017. Disponível em: https://cs.uwaterloo.ca/journals/JIS/VOL20/Bilgici/bilgici4.html. Acesso em: 2 out. 2019.

CATARINO, Paula. k-Pell, k-Pell-Lucas, modified k-pell sedenions, Asian European Journal of Mathematics. v. 12, n. 2, p. 1- 10. 2018. Disponível em: https://www.worldscientific.com/doi/pdf/10.1142/S1793557119500189. Acesso em: 2 out. 2019.

CERDA-MORALES, Gamaliel. Oresme polynomials and their derivatives. 2019. arXiv:1904.01165 [math.CO]. Disponível em: https://arxiv.org/abs/1904.01165. Acesso em: $1^{\text {o }}$ fev. 2019.

COOK, Charles. Some sums related to sums of Oresme numbers. In: HOWARD, Fredric T. Application of Fibonacci numbers. Dordrecht: Springer, 2004. v. 9.

HORADAM, A. F. Oresme numbers. The Fibonacci quarterlly, v. 12, n. 3, p. $267-271$, 1974. Disponível em: https://www.fq.math.ca/12-3.html. Acesso em: $1^{\circ}$ abr. 2019.

KAUERS, Manuel; PAULE, Peter. The concrete tetrahgedrom: symbolic sums, recurrence equations, generating functions, asymptotic estimates. New York: Springer, 2010.

NICODEMI, Olympia. Galileo and Oresme: Who is modern? Who is medieval? Mathematics Magazine, v. 83, p. 24-32, 2010. Disponível em: http://qrc.depaul.edu/djabon/CTTI/Readings/Nicodemi_Galileo_and_Oresme.pdf. Acesso em: 4 mar. 2019.

SOYKAN, Yüksel. Tribonacci and Tribonacci-Lucas sedenions. Mathematics. v. 7, n. 74, 2019. Disponível em: https://www.mdpi.com/2227-7390/7/1/74. Acesso em: 4 fev. 2019.

STILLWELL, John. Mathematics and it's history. New York: Springer-Verlag, 1989. 